\title{
Quantum dot-doped glasses and fibers: fabrication and optical properties
}

\section{Guoping Dong *, Haipeng Wang, Guanzhong Chen, Qiwen Pan and Jianrong Qiu*}

State Key Laboratory of Luminescent Materials and Device, School of Materials Science and Engineering, South China University of Technology, Guangzhou, China

Edited by:

Yuanzheng Yue, Aalborg University,

Denmark

Reviewed by:

Junjie Zhang, China Jiliang University, China

Danping Chen, Chinese Academy of Sciences, China

${ }^{*}$ Correspondence:

Guoping Dong and Jianrong Qiu,

State Key Laboratory of Luminescent

Materials and Device, School of

Materials Science and Engineering,

South China University of Technology,

Wushan Road 381, Tianhe District,

Guangzhou, Guangzhou 510640,

China

e-mail:dgp@scut.edu.cn:

qjr@scut.edu.cn
Quantum dot (QD)-doped glasses have been the hotspot for their excellent electronic and optical properties. Owing to its tunable and broadband near-infrared (NIR) emission by controlling the size and distribution of QDs, QD-doped glasses and fibers are potentially applied in photoelectric devices. In this review, we mainly introduce the preparation, tunable emission, and multi-wavelength optical amplification of QD-doped glasses. Due to their excellent optical performances, the fabrication of QD-doped glass fibers is also presented, containing the successful fabrication of QD-doped glass fibers with tunable NIR emission. Furthermore, the achievements and existing problems about QD-doped glasses and fibers are also proposed with several prospects. These QD-doped glasses and fibers show promising applications as the gain medium of NIR broadband fiber amplifiers and tunable fiber lasers.

Keywords: quantum dots, glass, fiber, tunable photoluminescence, optical amplification

\section{INTRODUCTION}

Quantum dots (QDs) with excellent electronic and optical properties have received significant development in the last decade. The quantum size effect allows QDs to exhibit great characteristic in optical and physical chemistry, and thus providing potential applications in diverse areas including lasers, saturable absorbers, bio-labels, light-emitting diodes (LED), and so forth (Guerreiro et al., 1997; Wise, 2000; Hines and Scholes, 2003; Hoogland et al., 2006; Pang et al., 2010; Salavati-Niasari et al., 2010; Sohila et al., 2011; Zhonghai et al., 2011).

Quantum dots are also known as semiconductor crystallites with a radius approximate equal to or less than their Bohr radii to constrain their carriers in three dimensions. Therefore, compared to bulk crystallite, QDs possess some unique quantum effects such as quantum confinement effect, size effect, surface effect, macroscopical quantum tunnel effect, splitting up the electronic structure of QDs from a continuous level into several separations (as shown in Figure 1). As a result, QDs are in a transition phase between particle and crystal that are completely different in bulk materials. For example, QDs will emit photonics at different wavelengths by tuning their size after activated. In other words, it can easily tune their energy band gap to control the absorption and photoluminescence (PL), as shown in Figure 2. Compared with the inherent and non-adjustable emissions of most rare earth RE ions, QDs can achieve tunable broadband emission in various regions, which can be used as the gain medium of broadband fiber amplifiers and tunable fiber lasers.

Quantum dots are based on the elements of group II-VI, III-V, IV-VI in the Periodic table. It is helpful to classify their structures into core (CdS and CdSe, $\mathrm{PbS}, \mathrm{PbSe}$ ), core/shell (CdS/ZnSe,
CdSe/CdS), and core/shell/shell (CdSe/CdS/ZnS, CdTe/CdS/ZnS) in terms of their composites (Kunets et al., 2004; Jacob et al., 2005; Thantu, 2005). QDs drew much attention for its unique optical feature in the earliest researches, resulting in intensive researches on photoelectric field (Jain and Lind, 1983). In 1980s, scientists focused more on mutual properties of QDs, such as quantum effect (Takagahara, 1987; Brus, 1991) and non-linear optics (Miller et al., 1988; Beenakker, 1991). QDs then showed great potential as fluorescent probes applying to biomedical field in the later development (Derfus et al., 2004; Kim et al., 2004; Michalet et al., 2005).

Combining the tunable optical properties of QDs with glass which is excellent transparent and easy-drawing, the QDs-doped glass and fiber have become popular in research (Feng et al., 2008). Besides, QDs-doped glasses are chemical and thermal stable with short response time as well as large three-order non-linear polarizability which is suggested to apply in optical communication. To fulfill the need of optical communication capacity, QDs-doped glass is most likely to become a glass fiber amplifier with a wider broadband, a higher amplification, and a lower noise. One QDs-doped glass amplifier can replace several existed amplifiers because of its tunable broadband PL and amplification. In addition, QDs-doped glasses are much likely to realize application in passive Q-switching and mode locking elements, including mode locking devices for Kerr-lens modelocked solid-state lasers (Malyarevich et al., 2000; Heck et al., 2007; Kuntz et al., 2007). Furthermore, QDs-doped glasses are great generators for short pulses activated by lasers operating in spectral ranges $0.7-0.9,1.06-1.08$, and $1.2-1.3 \mu \mathrm{m}$ and so forth (Melekhin et al., 2008). These potential and underway 

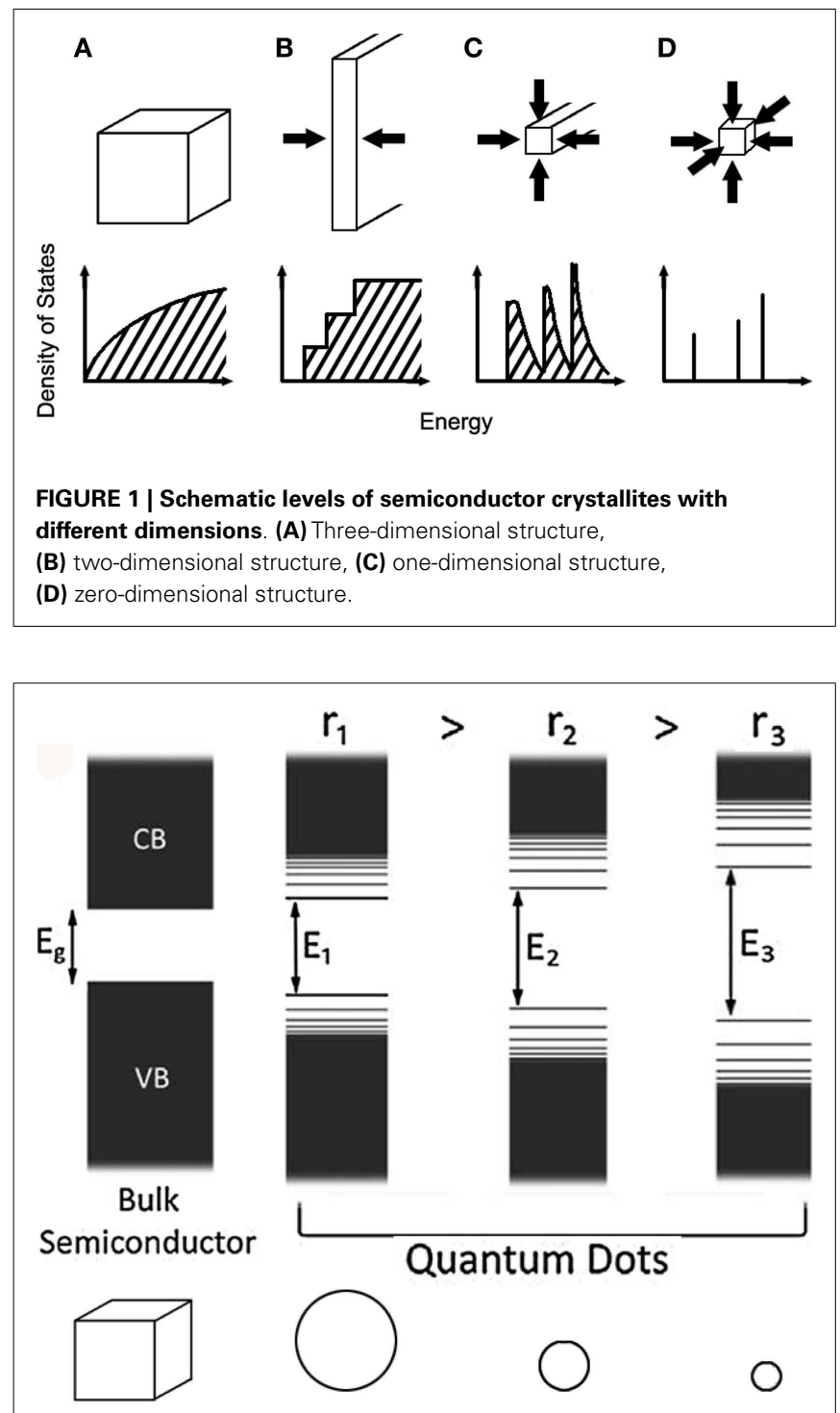

FIGURE 2 | Influence from the size of materials to its band gap

applications show a bright future of QDs-doped glass and fibers.

\section{OD-DOPED GLASSES \\ FABRICATION}

Various approaches have been developed to fabricate QDs-doped glasses including co-melting, sol-gel, ion-implantation, and so forth.

\section{Glass melt-quenching method}

Mixing and melting the composites of QDs precursor with the glass batch together, then a glass without QDs will be obtained. By certain process of nucleating, QDs will subsequently grow inside the glass. Borrelli and Smith used this method to fabricate QDsdoped glass as early as 1994 (Borrelli and Smith, 1994). Silva et al. successfully doped $\mathrm{S}$ into $\mathrm{SiO}_{2}-\mathrm{Na}_{2} \mathrm{CO}_{3}-\mathrm{Al}_{2} \mathrm{O}_{3}-\mathrm{PbO}_{2}-\mathrm{B}_{2} \mathrm{O}_{3}$-based

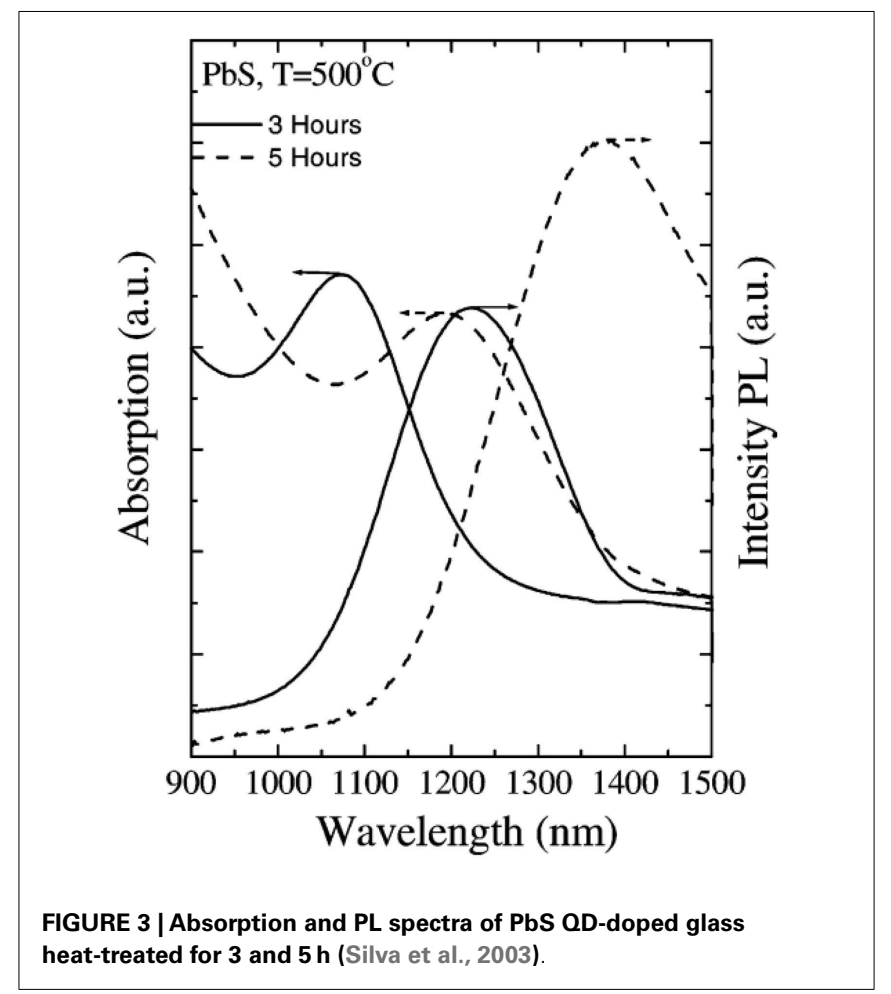

glass to grew PbS QDs after certain thermal treatment (Silva et al., 2003, 2006a). The absorption and PL spectra of PbS QD-doped glass heat-treated for 3 and $5 \mathrm{~h}$ are shown in Figure 3, respectively (Silva et al., 2003). It is clear that a red-shift occurred when the QD-doped glass was heat-treated for a longer period, which indicates that the size of QDs grows larger.

In our group, we used $\mathrm{SiO}_{2}-\mathrm{B}_{2} \mathrm{O}_{3}-\mathrm{K}_{2} \mathrm{O}-\mathrm{BaO}-\mathrm{ZnO}$ glass system as matrix to mix with $1-1.5 \mathrm{~mol} \% \mathrm{PbS}$ for the formation of $\mathrm{PbS} \mathrm{QDs}(\mathrm{P} 1, \mathrm{P} 1.5)$. In contrast, another system using $\mathrm{ZnS}-\mathrm{PbO}$ to indirectly produce $\mathrm{PbS}$ as the starting materials (Z2; Guoping et al., 2011). The as-prepared glasses were subsequently heat-treated at several temperatures to precipitate PbS QDs. Figure 4 shows the transmission electron microscope (TEM) images and size distribution of $\mathrm{PbS}$ QDs in representative $\mathrm{P} 1$ and $\mathrm{Z} 2$ glass samples, which suggest that using $\mathrm{ZnS}-\mathrm{PbO}$ to replace $\mathrm{PbS}$ as precursor of $\mathrm{PbS}$ QDs, the size distribution of PbS QDs in glasses becomes more uniform.

In Figure 5, broadband near-infrared (NIR) absorption and emission peaks are observed in the spectra, which is due to the recombination of electron and hole pairs created by the excitation source. With the enhanced heat-treatment temperature, PbS QDs grow larger, which will result in the red-shift of PL emission band. The size distribution of PbS QDs in glasses also becomes wider.

Although the method is convenient to prepare QDs-doped glasses, it requires particular high temperature to melt raw materials. Several other groups also developed other low temperature routines to fabricate QD-doped glasses. Several glass systems like tellurite and phosphate glasses are developed to lower the melting temperature, but they usually have a lower mechanical property than silica glasses (Kolobkova et al., 2002; Savitski et al., 2002; Jacob et al., 2005). 

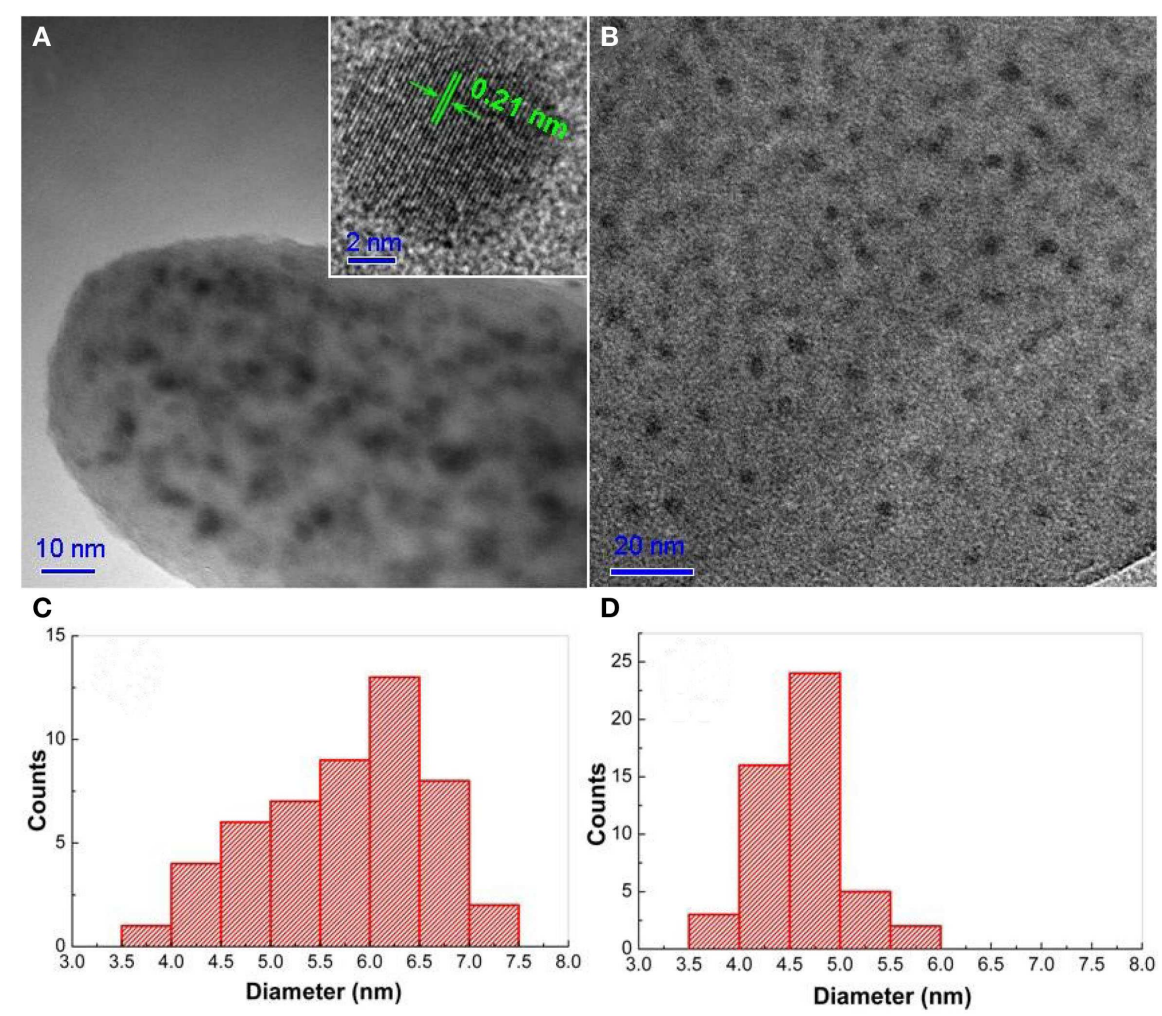

FIGURE $\mathbf{4}$ |TEM image of (A) P1 glasses and (B) Z2 glasses heat-treated at $\mathbf{5 8 0}{ }^{\circ} \mathbf{C}$ for $\mathbf{2 4} \mathbf{h}$. The insert of (A) shows the HR-TEM image of a single PbS OD. (C,D) Show the size distribution of PbS QDs in P1 and Z2 glasses corresponding to TEM image (A,B), respectively (Guoping et al., 2014).

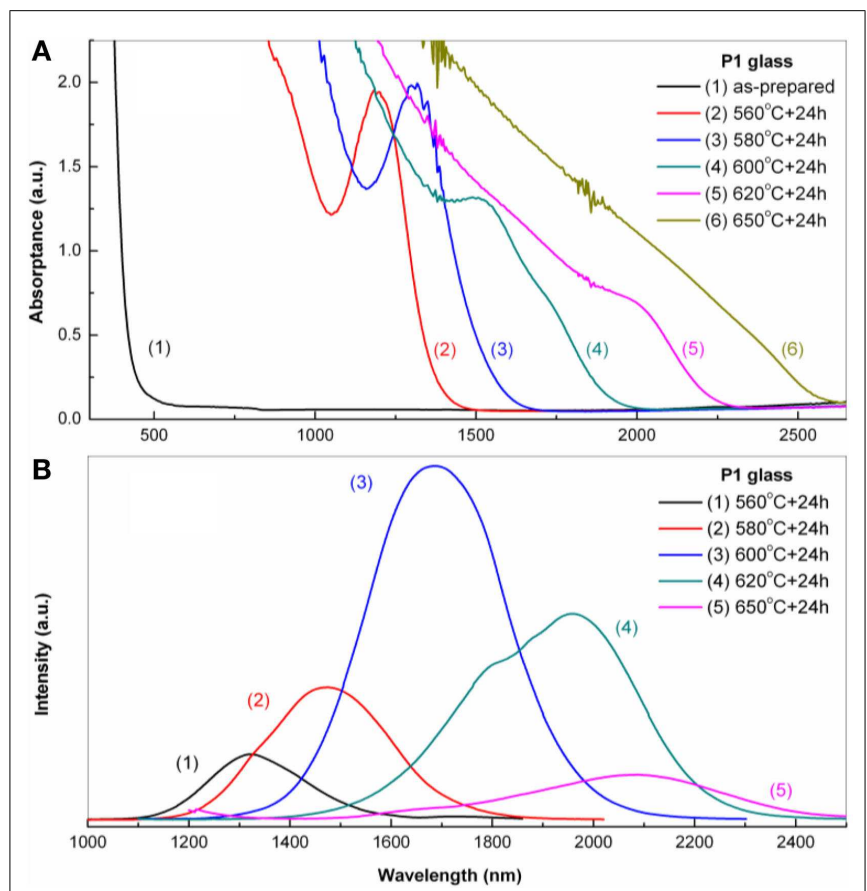

FIGURE 5 | (A) Absorption and (B) PL spectra of P1 glass heated at different temperature for $24 \mathrm{~h}$ (Guoping et al., 2011; Huang et al., in press).

\section{Sol-gel method}

Colloidal QDs systems including $\mathrm{CdS}, \mathrm{ZnS}, \mathrm{PbS}$, and $\mathrm{ZnO}$, have been successfully reported in many previous investigations (Bahnemann et al., 1984; Henglein, 1988; Kormann et al., 1988). Since Rajh et al. utilized a sol-gel method to incorporate QDs in transparent silicate glasses conveniently at low temperature (Rajh et al., 1988), sol-gel method is now considered as a reliable way to produce QD-doped glass (Planelles-Arago et al., 2008; Mashford et al., 2011). Planelles-Aragó's group reported highly transparent and colorless silica embedded with $\mathrm{Eu}^{3+}$-doped $\mathrm{ZnS}$ QDs (Planelles-Arago et al., 2008). In their works, the solution of zinc nitrate and europium nitrate were first dissolved in DMSO [dimethyl sulfoxide, $\left(\mathrm{CH}_{3}\right)_{2} \mathrm{SO}$ ] and then stirring for $30 \mathrm{~min}$. The resulting solution subsequently added to the mixture of TEOS [tetraethoxysilane, $\mathrm{Si}\left(\mathrm{OC}_{2} \mathrm{H}_{5}\right)_{4}$ ], DMSO, and water. After stirring and oven dry, the transparent and colorless xerogels were fired in a furnace at $500^{\circ} \mathrm{C}$, and finally formed a QD-doped glass. The TEM images in Figure 6 show that typical spherical ZnS QDs were embedded in the amorphous silica medium. Emission spectra in Figure 7 illustrate a broad blue emission of $\mathrm{ZnS}$ QDs and red ${ }^{5} \mathrm{D}_{0} \rightarrow{ }^{7} \mathrm{~F}_{2}$ transition $(615 \mathrm{~nm})$ emission of $\mathrm{Eu}^{3+}$ ions, respectively. Both the TEM and emission results confirmed that QDs were successfully doped into the glass matrix by sol-gel method.

Compared to melt-quenching, the sol-gel route is helpful to lower the melting temperature and disperse the precursors 


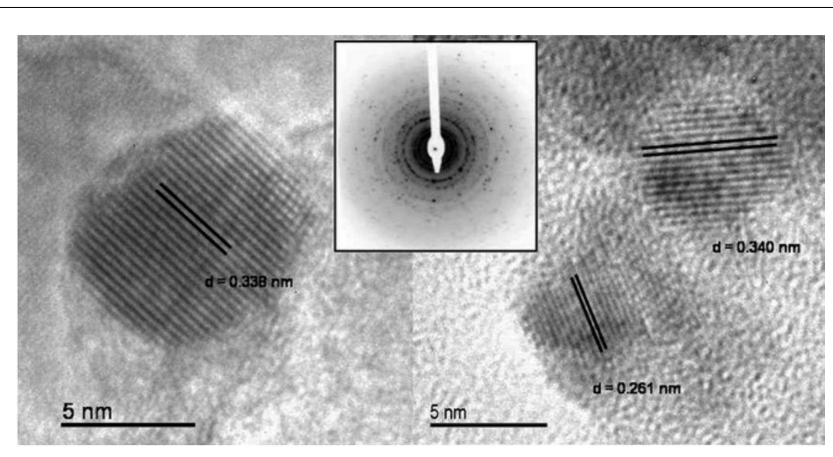

FIGURE 6 | High resolution-transmission electron microscopy (HR-TEM) micrographs and selected area electron diffraction (SAED) patterns (insert) of $\mathrm{Eu}^{3+}$-doped $\mathrm{ZnS}$ (EZS) sample after firing at $500^{\circ} \mathrm{C}$ (Planelles-Arago et al., 2008).

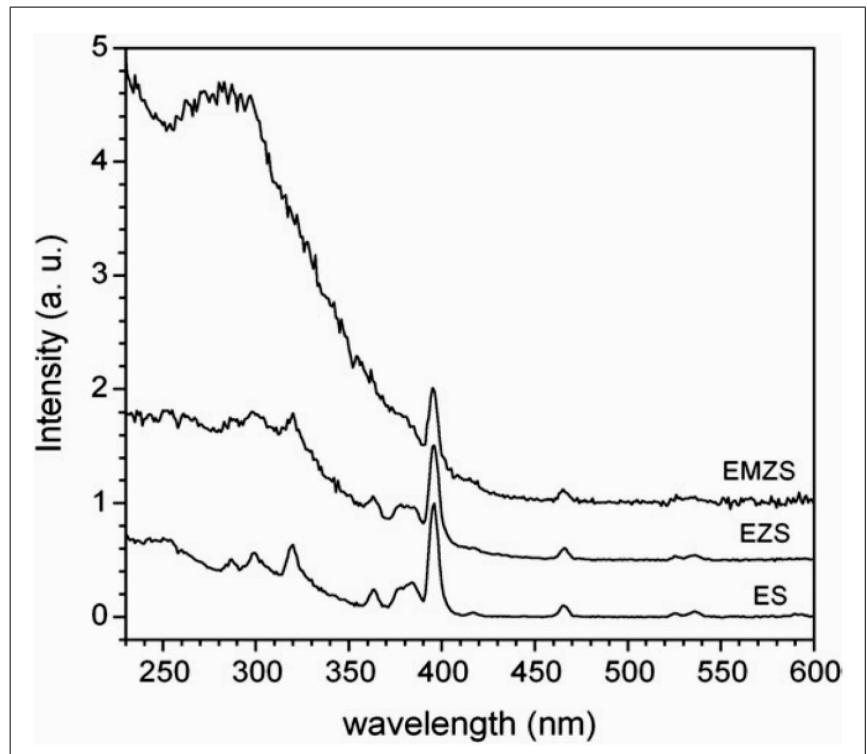

FIGURE 7 | Emission spectra of Eu ${ }^{3+}$-doped (EZS) and $\mathrm{Eu}^{3+}$, $\mathrm{Mn}^{2+}$-co-doped (EZMS) $\mathrm{ZnS}-\mathrm{SiO}_{2}$ samples annealed at $500^{\circ} \mathrm{C}$ upon excitation at $280 \mathbf{n m}$. $\mathrm{Eu}^{3+}$-doped $\mathrm{SiO}_{2}(\mathrm{ES})$ and $\mathrm{ZnS}_{-} \mathrm{SiO}_{2}(\mathrm{ZS})$ have been included as references (Planelles-Arago et al., 2008).

homogeneous easily. However, fabrication in large bulk material is still a challenge because the residual stress is hard to eliminate. Therefore, the sol-gel method is only suitable for QDs-doped thin films. In addition, the difficult removal of organic ligands introduced by synthesis procedure and the restricted silica glass matrix may limit the development of sol-gel route.

\section{Ion-implantation method}

Ions accelerated by electric field are injected into surface of the material. Ions implanted will react with the material, creating defect or forming composite. This method was originally used for semiconductor doping, then developed into a wider range of applications in the preparation of integrated circuits and modification of metals. Shortly after, there were numerous researches about the effects of implantation on silica matrix (Primak and Kampwith, 1968; Arnold, 1973; EerNisse, 1974). Glasses implanted with various ions were begun to study afterward (Arnold, 1975; Mattern et al., 1976). Ion-implantation is mainly used to nanocomposites like metallic nanocrystals, semiconductor nanocrystals, and ferromagnetic nanoparticles (Meldrum et al., 2001). After decades of investigation, ion-implantation still draws high attention (Lee et al., 2005; Carder et al., 2013). In the work conducted by Carder's et al., slides of $\mathrm{SiO}_{2}$ was used as a substrates, which were ion-implanted with $\mathrm{Pb}(29 \mathrm{keV})$ and Se $(25 \mathrm{keV})$ ions under vacuum at $5 \times 10^{-8}$ mbar (Carder et al., 2013). The samples were then transferred to an electron beam annealing (EBA) system and maintained at a temperature around 760-915 $5^{\circ}$.

The TEM images confirm that PbSe QDs have been successfully implanted into the silica matrix (Figures 8A,B), and there are a band of nanoclusters at about $50-70 \mathrm{~nm}$ beneath the surface (Figure 8A). Figure 9 shows the PL spectra of samples applied to different annealing process, indicating that QDs embedded inside the glass can be further tuned by EBA temperature.

Although ion-implantation can induce the formation of tunable QDs beneath the surface of glass matrix, the ions implanted are limited around the surface, and barely reach the inner part. Therefore, it is a challenge to produce scaling QDs-doped bulk glasses.

Sol-gel route is the most convenient protocol to synthesis QDs with excellent performance. However, for further application, QDs must be incorporated into various bulk matrixes. Ionimplantation is able to synthesize all kinds of QDs-doped glasses, the shallow implantation depth of QDs beneath the matrix surface limits its further applications. In spite of high temperature and relatively complex procedure, melting-quenching is a high prior to synthesize a QDs-doped glass due to the controllable size and distribution of QDs in various glass matrixes.

There are also some other methods like atomic layer epitaxy (ALE; Mukai et al., 1997), molecular beam epitaxy (MBE; Grundmann et al., 1995; Mirin et al., 1995), metal-organic chemical vapor deposition (MOCVD; Heinrichsdorff et al., 1997; Tan et al., 2005), and aqueous synthesis (Rogach et al., 2007), which are relatively complex and less used. Therefore, they are not discussed in detail here.

\section{OPTICAL PROPERTIES \\ Tunable optical properties}

Since the quantum confinement of QDs first observed by Efros and Ekimov (Efros and Efros, 1982; Ekimov et al., 1985), Brus modeled the excited electronic state theoretically in order to understand the redox potential that depends upon size (Brus, 1984). Later on, Henglein et al. successfully synthesized QDs with different sizes (Weller et al., 1984; Spanhel et al., 1987), which create a precedent in the research field.

Energy spectrum of electrons in a bulk material is almost continuous, therefore, electrons in fact is free (Figure 1A). However, when the material gets smaller, electrons will generally be restricted and exhibit a discrete energy spectrum in macro (Figures 1B-D). Especially when a particle's size tend to the Bohr radii of the bound state of an electron and a hole, the movement of electrons is 

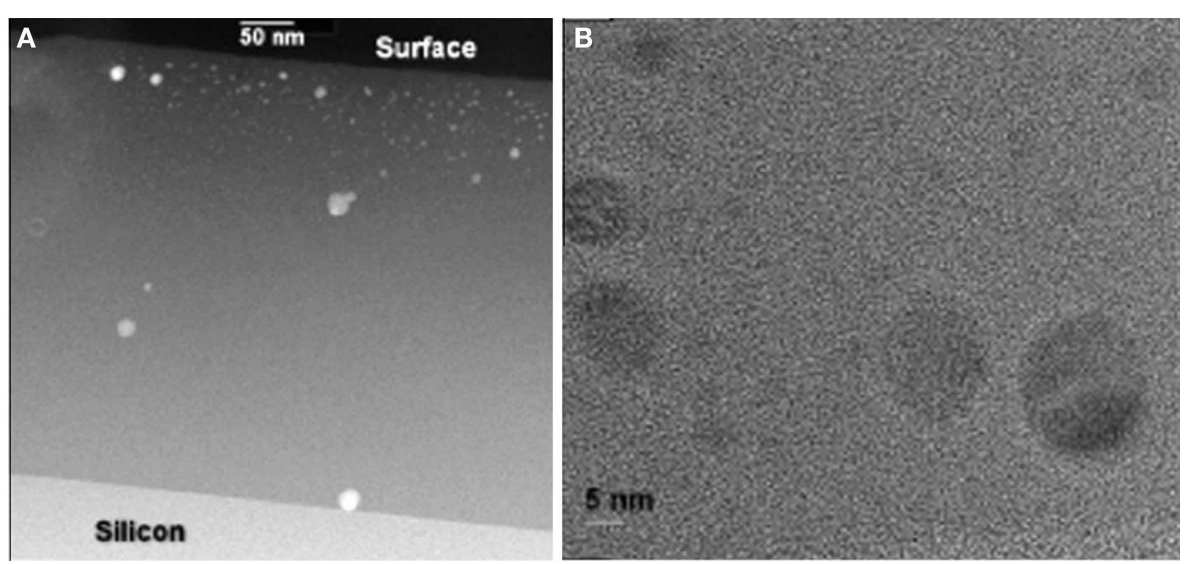

FIGURE 8 | (A) High-angle annular dark field and scanning transmission electron microscopy (HAADF-STEM) cross-sectional image of the Pb+Se implanted and annealed sample. (B) Cross-sectional TEM image of a number of PbSe clusters in the near-surface region (Carder et al., 2013).

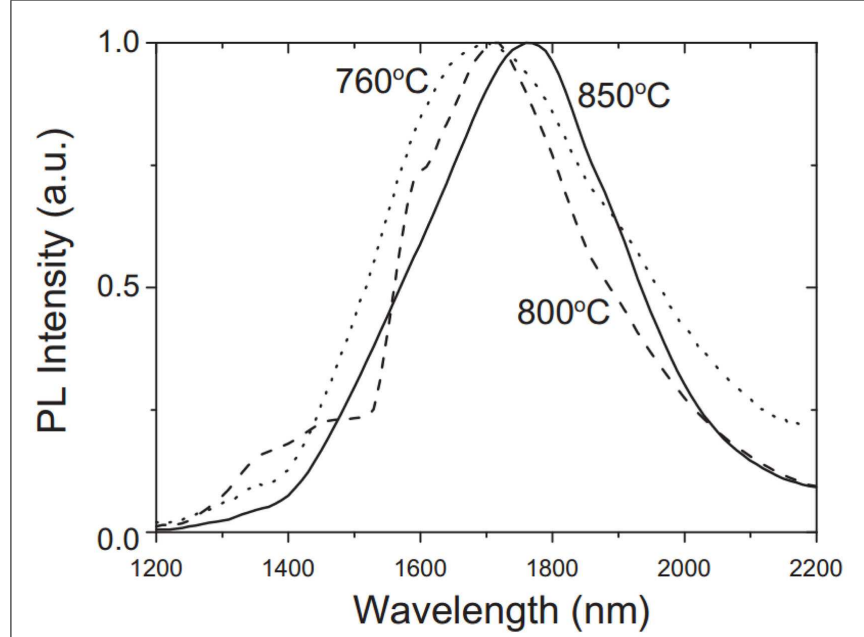

FIGURE 9 | Normalized low temperature (4 K) PL spectra from samples electron beam annealed at 760,800 , and $850^{\circ} \mathrm{C}$, respectively (Carder et al., 2013).

strongly confined, lending to a unique tunable optical property to QDs.

Due to the quantum confinement effect, the band gap of electrons depends upon their sizes. In other words, QDs get smaller, their band gaps become wider. It offers a convenient way to tune the QDs absorption and PL wavelength by controlling their size.

Work of Murray et al. can show what above more directly: they synthesize $\mathrm{CdE}(\mathrm{E}=\mathrm{S}, \mathrm{Se}, \mathrm{Te})$ from $\sim 12$ to $\sim 115 \AA$ in diameter by sol-gel method (Murray et al., 1993). The series spans a range of sizes from nearly molecular species to bulk lattice. An obvious redshift can be observed in Figure 10 with the size of CdSe QDs getting larger, which matches well with the theory mentioned above. As Figure 11 illustrated, transition energy of CdSe crystallites as a function of size is compared with the prediction of the effective mass approximation. Experiment and theory fit well when the size of QDs is larger enough, but it does not in the case of small size

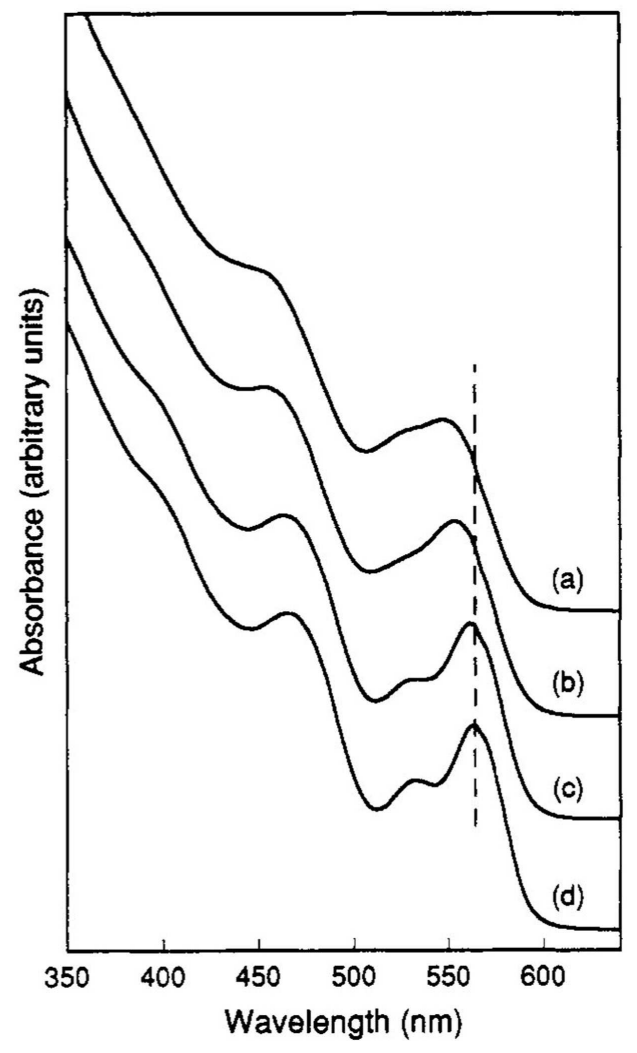

FIGURE 10 | Room temperature optical absorption spectra of CdSe nanocrystallites dispersed in hexane and ranging in size from $\sim 12$ to $\sim 115$ Å (Murray et al., 1993).

because of the non-parabolicity of the bands at higher wave vectors and the finite potential barrier at the surface of the particles.

During the last decade, synthesis of QDs have been improved and further expanded to control the QDs' morphology and structure (Peng et al., 1998; Manna et al., 2000; 


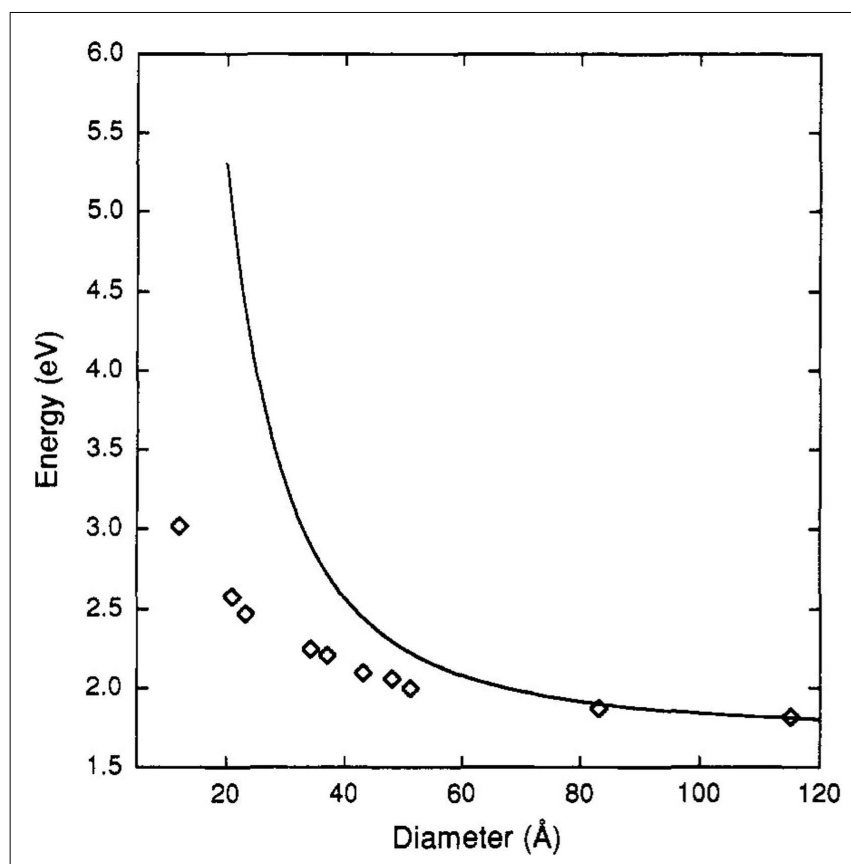

FIGURE 11 | Transition energy of CdSe crystallites as a function of size (diamonds) compared with the prediction of the effective mass approximation (solid line; Murray et al., 1993).

Peng and Peng, 2001a). Figure 12 shows the influences of CdSe QDs' shape and structure on their optical properties: (1) A wider splitting between absorbing and emitting states was shown in rods than that in dots (Figures 12A,B); (2) quantum yields rises at first and goes down latter with the increasing thickness of a shell (CdS or $\mathrm{ZnS}$ ) grown on the CdSe core (Figure 12C); (3) polarization anisotropy can be observed from quantum rod while QD cannot (Figure 12D).

All examples above only focused on the tunable optical properties of QDs themselves. However, for better application, controlling QDs in glasses is more important. Heo and his coworkers have successfully controlled the size of $\mathrm{PbS}$ QDs using rare earth ions and $\mathrm{Ag}^{+}$ions (Heo and Liu, 2007; Chao and Jong, 2014; $\mathrm{Xu}$ and Heo, 2014). Take RE ion for example: the size of QDs decreased with RE ion concentration increases under the same heat-treatment conditions and thereby provided another pathway for the controlled formation of QDs (Chao and Jong, 2014). Their group also synthesized glasses doped with different percents of $\mathrm{Nd}_{2} \mathrm{O}_{3}$, and their absorption and PL spectra are shown in Figure 13. The peak wavelength of the first absorption bands shifted from 1415 to $1274 \mathrm{~nm}$ as $\mathrm{Nd}_{2} \mathrm{O}_{3}$ content increased from 0 to $0.6 \mathrm{~mol} \%$ under the same heat-treatment (Figure 13A). Emission peak wavelength of the PL bands also shifted from 1516 to $1370 \mathrm{~nm}$ at the same time (Figure 13B).

In addition to the quantum confinement, some external field factors, such as temperature, pump intensity, can also influence QDs' optical properties (Olkhovets et al., 1998; Schaller et al., 2003). Olkhovets et al. observed that the temperature coefficients of electron-hole pair energies $(d E / d T)$ in $\mathrm{PbS}$ and PbSe QDs depend strongly on their size (Olkhovets et al., 1998), resulting
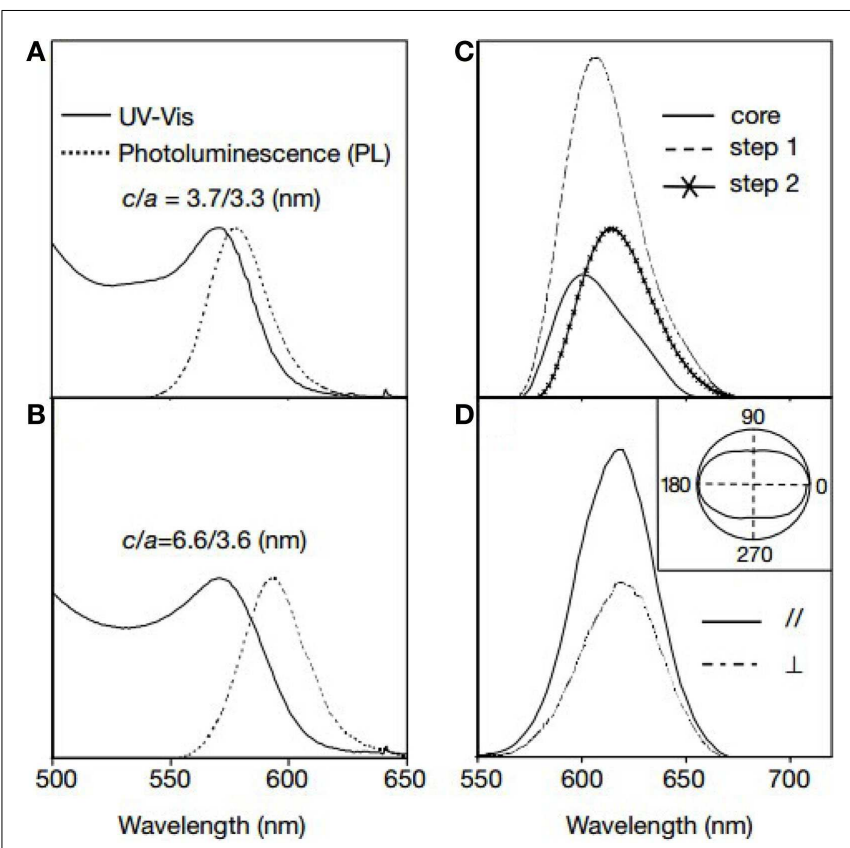

FIGURE 12 | Influences of shape on the optical properties of CdSe QDs (A) Ultraviolet-visible (UV-Vis) and photoluminescence (PL) spectra of CdSe QDs. (B) UV-Vis and PL spectra of CdSe QDs. (C) PL spectra of core/shell QDs with different shell thickness. (D) Polarization resolved PL spectra of CdSe QDs (Xiaogang et al., 2000).

in the special electronic and vibrational properties in QDs (Wise, 2000). The experimental data are shown in Figure 14. It is clear induced that the absorption spectra of smaller QDs are almost independent of temperature while the larger ones strongly depend on the ambient temperature.

Schaller and his coworkers also demonstrated that the amplified spontaneous emission (ASE) from PbSe QDs with emission energies tunable in the NIR (Schaller et al., 2003). They synthesized $\mathrm{PbSe}$ QDs by sol-gel method to ensure a good surface passivation and high filling factor. An emission band at $1555 \mathrm{~nm}$ is observed when the pump intensity increased (Figure 15), showing that $\mathrm{PbSe}$ QDs have a strong dependence on the pump intensity.

By achieving tunable optical properties, people can obtain QDs in glass with multi-wavelength NIR emission. And it is the first step to obtain tunable NIR fiber amplifiers and lasers. In the next section, we will discuss about the optical amplification of QDs-doped glasses. Combing their tunable NIR emission with the optical amplification, the NIR broad amplifiers and tunable lasers will have a bright future.

\section{Optical amplification}

Since the CdSe QDs were first demonstrated to be available in the visible optical amplification (Klimov et al., 2000), nowadays, the wavelength of optical amplification have explored from visible to infrared. For infrared broadband PL semiconductor QDs, the research system is mainly about $\mathrm{PbS}$, InAs, $\mathrm{PbSe}, \mathrm{HgTe}$, and so forth (Steiner et al., 2004; O'Connor et al., 2005; Sukhovatkin et al., 2005). Among them, PbS QDs with a large Bohr exciton radii $(\sim 18 \mu \mathrm{m})$ and a moderate band gap at near/mid-infrared 

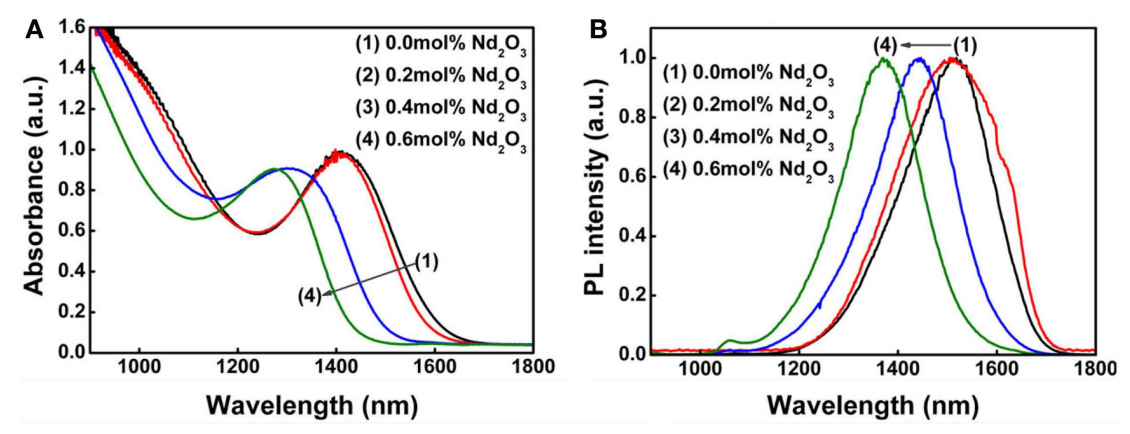

FIGURE 13 | (A) Absorption and (B) PL spectra of $\mathrm{Nd}_{2} \mathrm{O}_{3}$ QD-doped glasses heat-treated at $500^{\circ} \mathrm{C}$ for $25 \mathrm{~h}$ ( $\mathrm{Chao}$ and Jong, 2014 ).
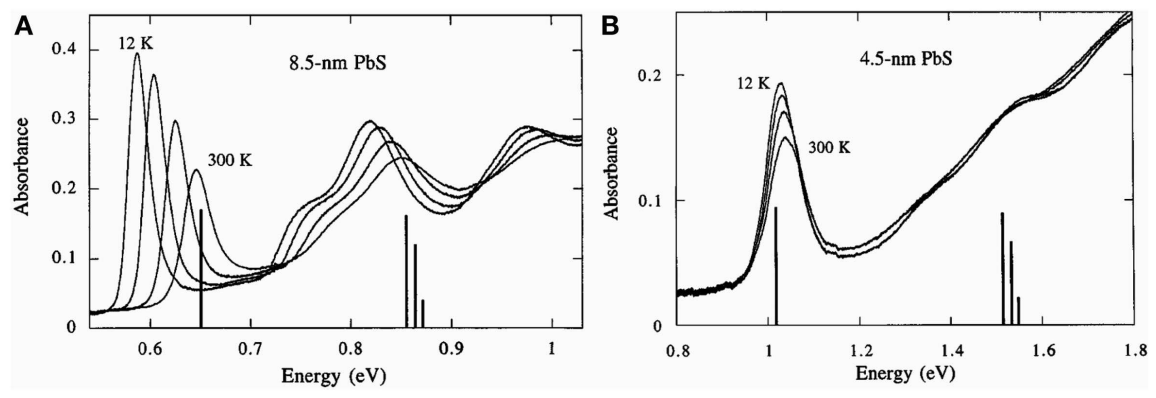

FIGURE 14 | Absorption spectra of $8.5 \mathrm{~nm}(\mathrm{~A})$ and $4.5 \mathrm{~nm}$ (B) PbS QDs recorded at 12, 100, 200, and 300 K (Olkhovets et al., 1998).

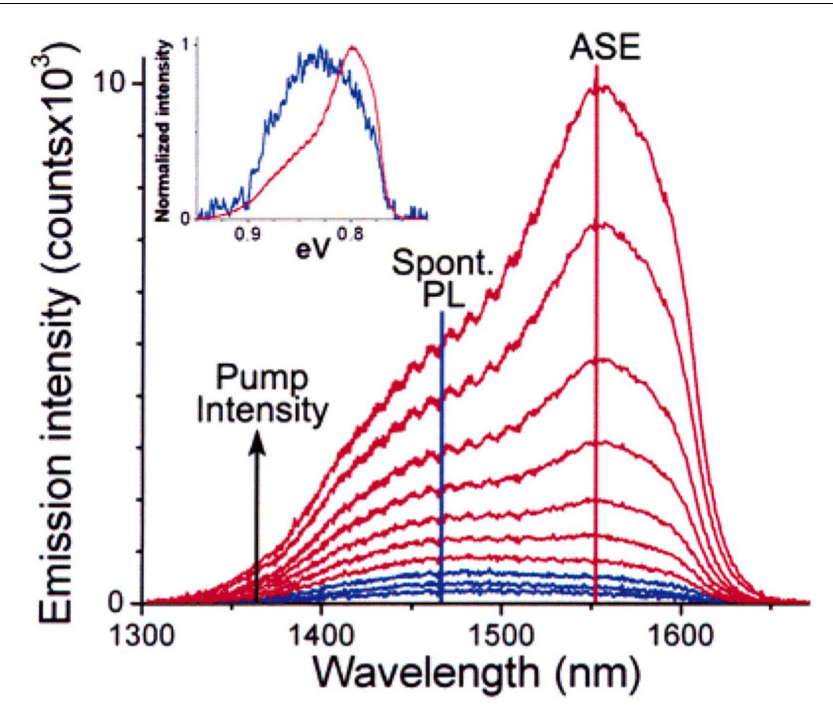

FIGURE 15 | Development of a spectrally narrowed ASE band at $\mathbf{1 5 5 5} \mathbf{\mathrm { nm }}$ is observed as a function of pump power for PbSe QDs Comparison of normalized emission spectra produced using low (blue) and high (red) pump intensities (inset), which are dominated by spontaneous PL and ASE, respectively, shows the ASE as a spectrally narrowed, red-shifted band (Schaller et al., 2003).

wavelength have gained great attention as NIR broadband amplified medium (Sargent, 2005; Heo and Liu, 2007; Chao et al., 2008).
In fact, $\mathrm{PbS}$ QDs were precipitated in glasses and a gain of $80^{-1} \mathrm{~cm}$ at $1300 \mathrm{~nm}$ was obtained at room temperature (Wundke et al., 1999). For further research, Huang et al. reported the tunable absorption and PL of PbS QDs in the glasses precipitated through thermal treatment (Huang et al., 2008). As shown in Figure 16, optical amplification at $1300 \mathrm{~nm}$ is observed, and the ASE spectrum is also measured to confirm the optical gain from $\mathrm{PbS}$ QDs.

Recently our group first demonstrated that PbS QDs-doped glasses exhibit two optical amplifications at the traditional $\sim 1330$ and $\sim 1550 \mathrm{~nm}$ optical communication windows, which is helpful to expand the applications of QDs in optical communication (Guoping et al., 2011). In order to further evaluate the potential of PbS QDs-doped glass applied in a broadband fiber amplifier, it is necessary to investigate the broadband optical amplification at several optical communication windows. We successfully obtained the optical amplification at both 1330 and $1550 \mathrm{~nm}$ simultaneously in single PbS QDs-doped glass (as shown in Figure 17) by tuning the QDs' size, distribution, and PL properties (Guoping et al., 2014). Our studies have confirmed that PbS QDs-doped glasses are promising to be applied as the gain medium of broadband fiber amplifiers and tunable fiber laser at optical communication windows.

Our studies also demonstrated that utilizing $\mathrm{ZnS}+\mathrm{PbO}$ instead of $\mathrm{PbS}$ as the precursor of QDs can effectively inhibit sulfur volatilization during glass melting, leading to the uniform and controllable growth of PbS QDs in glass matrix. From the optical gain spectra in Figure 18, it can be deduced that using $\mathrm{ZnS}+\mathrm{PbO}$ as precursor in $\mathrm{PbS} \mathrm{QD}$-doped glasses, the optical gain and its 
slope value (optical gain vs. pumping power) improve substantially (Huang et al., in press).

Herein, QD-embedded glass with multi-wavelength optical amplification is promising as the gain medium of broadband fiber amplifiers and tunable fiber lasers.

\section{QD-DOPED GLASS FIBER}

Based on the excellent broadband optical tunability of QD-doped glasses, it is necessary to draw them into fibers. In the following section, we will discuss the fabrication and optical properties of QD-doped glass fibers.

Quantum dots will show a secondary growth during the fiberdrawing process, therefore low temperature is necessary to fabricate QDs-doped glass fiber. A uniform QDs solution was filled into a hollow optical fiber from front to back, after which by curing, the

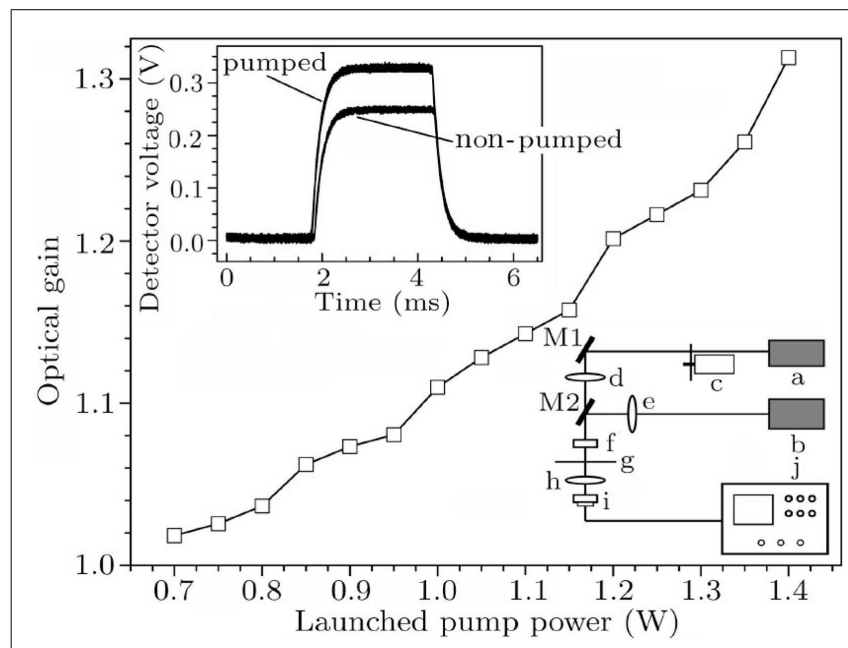

FIGURE 16 | Relation between the change in optical signal $I / I_{0}$ and the launched pumped power. $/$ and $I_{0}$ are the signal intensity with and without $980-\mathrm{nm}$ excitation displayed by digital oscilloscope. The bottom right of the inset shows the schematic diagram. The top left corner of the inset shows an oscilloscope image of the optical signal change at $1300 \mathrm{~nm}$ pumped with 980-nm laser diode (the power is 1.4W; Huang et al., 2008).
QD-doped fiber was successfully produced. Hreibi et al. reported the fabrication and characterization of a PbSe QDs liquid-core fiber (Hreibi et al., 2010). The PbSe QDs were synthesized based on the literature method (Yu et al., 2004). The liquid-core optical fiber was made by a pure silicate capillary waveguide with an outer $120 \mu \mathrm{m}$ and a core $17-\mu \mathrm{m}$ diameter. A $20-\mathrm{cm}$-long piece of fiber was immersed in the liquid medium containing PbSe QDs dispersed in toluene after $10 \mathrm{~min}$ to completely fulfill the core by capillary attraction (see Figure 19). Upon pumping by a 532nm-CW laser, a good ASE was efficiently transmitted with a peak centered at $1290 \mathrm{~nm}$ (see Figure 20).

Cheng et al. reported a PbSe QD-doped fiber laser (QDFL) with a ring resonator using colloidal $\mathrm{PbSe} \mathrm{QDs}$ as gain media in the background of UV curing adhesive (Cheng et al., 2013). Firstly, the UV curable adhesive solution containing the QDs with required

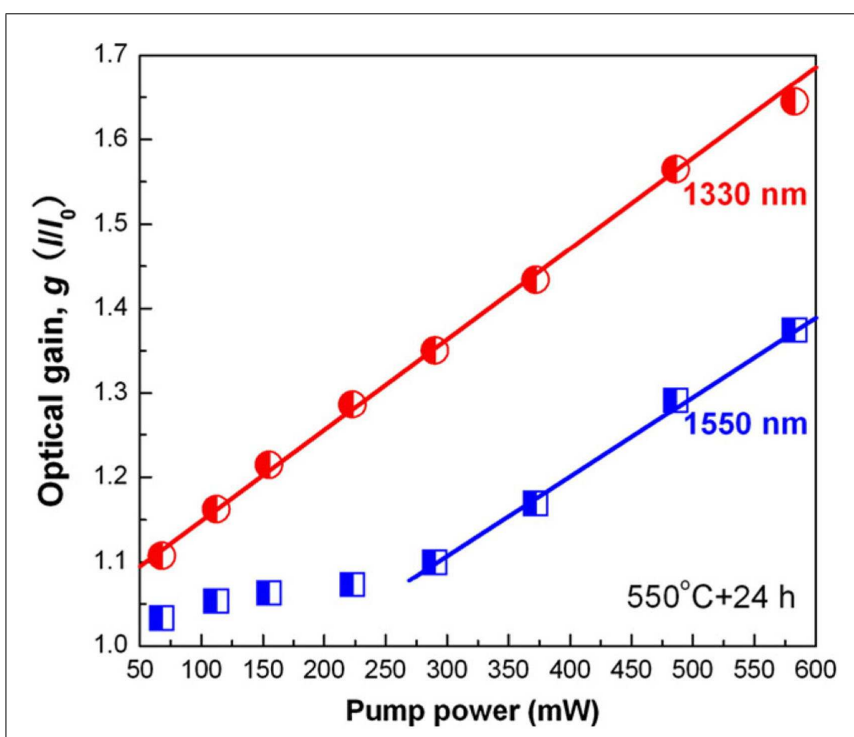

FIGURE 17 | Optical gains $\left(I / I_{0}\right)$ at $1330 \mathrm{~nm}$ (red) and $1550 \mathrm{~nm}$ (blue) of $\mathrm{PbS}$ OD-doped glass heated at $550^{\circ} \mathrm{C}$ for $24 \mathrm{~h}$, which are collected as a function of pumping power (Guoping et al., 2014).
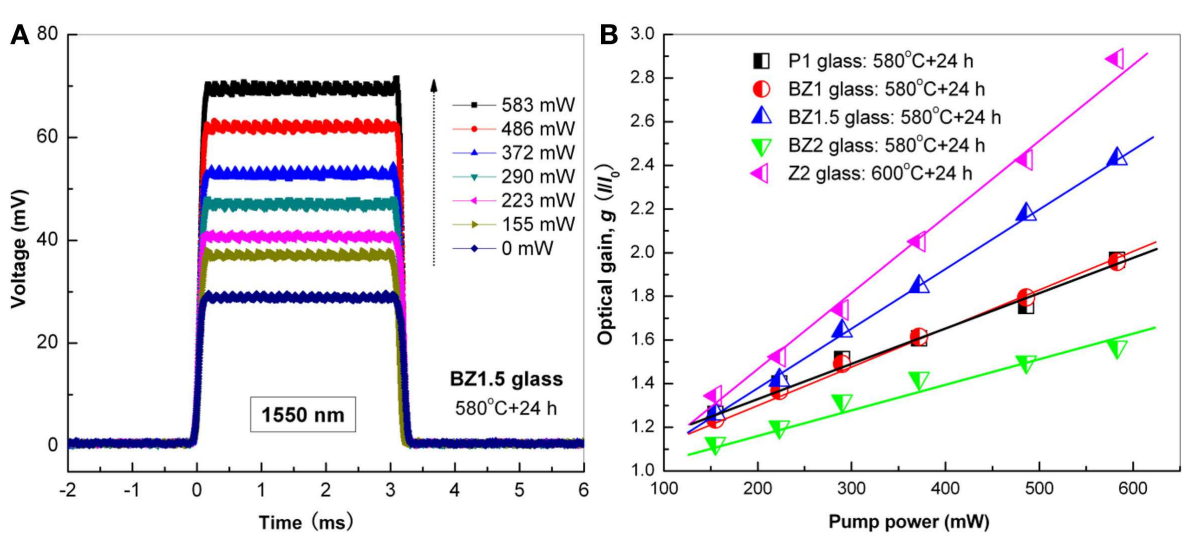

FIGURE 18 | (A) The amplified signals at $1550 \mathrm{~nm}$ of PbS QD-doped glass heated at $580^{\circ} \mathrm{C}$ for $24 \mathrm{~h}$. (B) Optical gain $\left(/ / / I_{0}\right)$ at $1550 \mathrm{~nm}$ of PbS QDs-doped glasses collected as a function of pumping power (Huang et al., in press). 


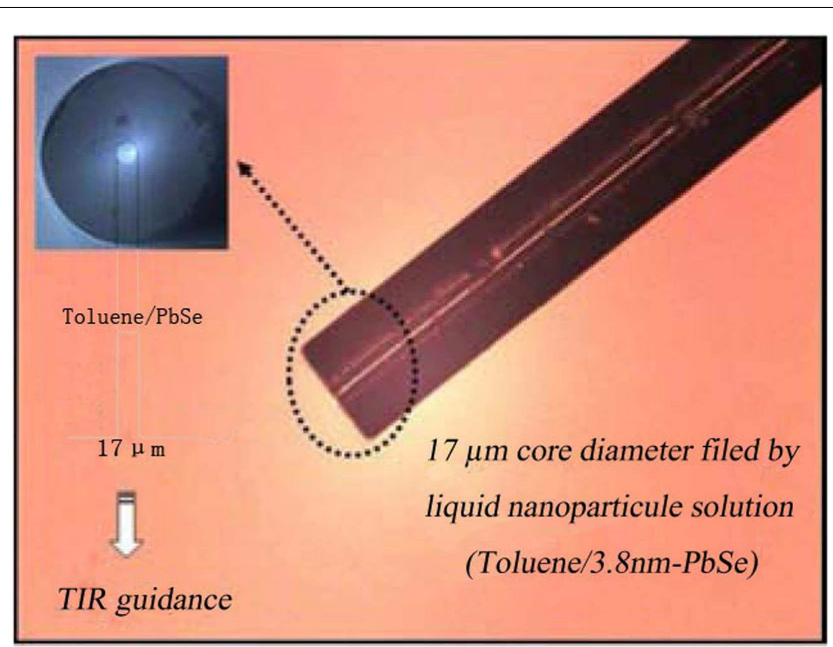

FIGURE 19 | Longitudinal view of a $17-\mu \mathrm{m}$ core diameter of a silica capillary filled by liquid nanoparticles solution (Hreibi et al., 2010).

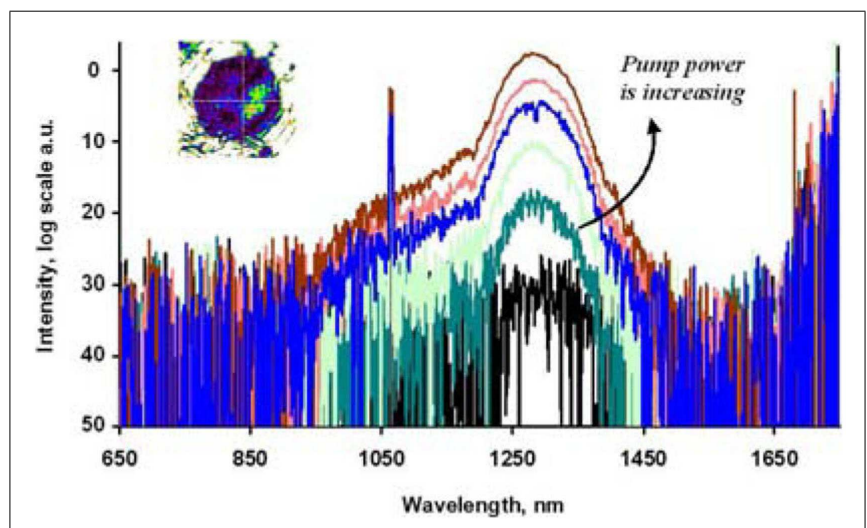

FIGURE 20 | Evolution of the spectra recorded at the output of the PbSe QDs liquid-core fiber for varying input power. Inset: near field pattern recorded at the output of the fiber (Hreibi et al., 2010).

concentrations were prepared. The resulting solution was then filled into a hollow fiber (50/125 $\mu \mathrm{m}$, core/cladding) at difference pressure. Subsequently, the PbSe QD-doped fiber was irradiated under a UV lamp for $\sim 15 \mathrm{~min}$ for curing the UV adhesive in fiber. The available QD-doped fiber with a tail was finally prepared. Upon 980-nm pump, a clear exciting threshold was recorded, and continuous yet stable laser oscillation at $1550 \mathrm{~nm}$ was observed for the first time. From the results in Figure 21, it can be known that when the pumping power is up to a certain level, the output power appears and linearly increases with the pumping power. These works could be a first step toward the development of a new family of fiber amplifiers and lasers.

Using the above QDs filling hollow glass fiber method to prepare QD-doped glass fiber, the fiber core substrate material should have specific requirements for their refractive index, transmission wave band, gel curing rate, and characteristic temperature. The

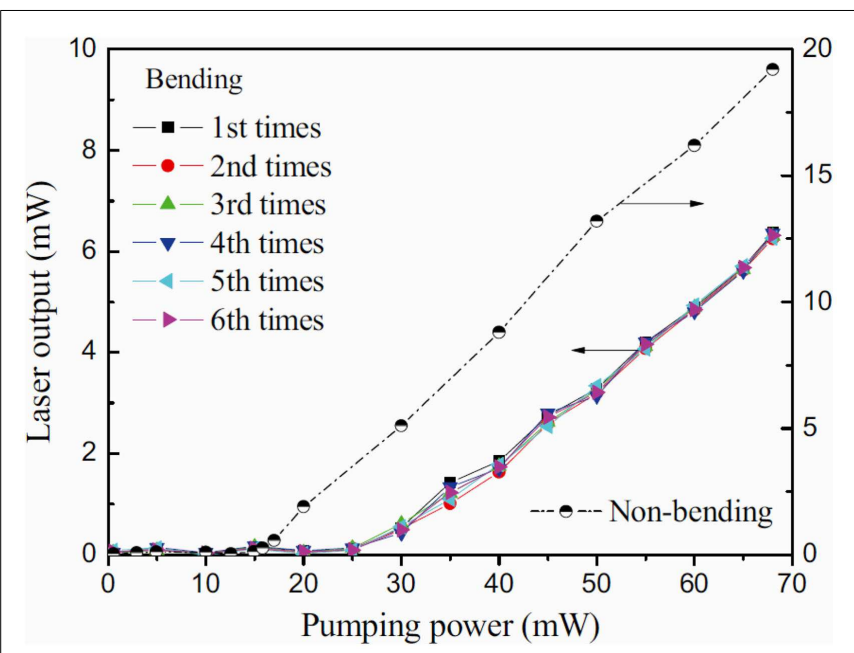

FIGURE 21 | Variation of the laser output with the pumping power inputted into the PbSe QD-doped fiber (Cheng et al., 2013).

above process is too complicated and difficult for large-scale promotion to use. Furthermore, the core substrate material is usually photocurable adhesive, which is easy to decompose under laser pump and output. It results in the poor photostability of the above QD-doped glass fiber. Therefore, directly drawing fiber preform is an alternative way to controllable fabricate QDs-doped glass fiber.

Fabrication of solid core fibers containing $\mathrm{PbS}$ QDs was attempted using the conventional fiber-drawing technology by Liu et al. (Chao and Jong, 2013). PbS QD-doped glass preform with a diameter of $1 \mathrm{~cm}$ and length of $10 \mathrm{~cm}$ was prepared by meltquenching method (Figure 22A). When heated up to $800^{\circ} \mathrm{C}$ for the fiber-drawing, the glass rod turned into black indicating that $\mathrm{PbS}$ QDs were formed during fiber-drawing process (Kellermann et al., 2001). The TEM image of the fiber showed the isolated small $\mathrm{PbS}$ QDs have a diameter of 4-6 $\mathrm{nm}$. However, most of the $\mathrm{PbS}$ nanocrystals were in the size of 30-60 nm (Figures 22B,C). Therefore, it is difficult to obtain intense NIR tunable emission.

Recently, our group also prepared the bulk PbS QD-doped glass preform, and drawn the QD-doped glass fiber by the fast drawing technique. This method successfully prepared a brown, transparent, chunk glass preform (Figure 23A). However, due to the uncontrollable secondary growth during fiber-drawing process, the glass fiber turned into black and light tight (Figure 23B). Most of the $\mathrm{PbS}$ nanocrystals grew larger than their exciton Bohr radius. Therefore, only weak NIR emission was detected in the PbS QDdoped glass fiber prepared by the above fiber-drawing. Based on the above results, a new modified fiber-drawing technique should be developed to prepare high-quality QDs-doped glass fibers.

To obtain intense NIR emission from PbS QD-doped glass fibers, we recently tried to prepare the bare glass fiber directly by melt-extraction method. After the glass melting process, the melt will be directly drawn into glass fiber at high temperature. After heat-treatment, glass fiber with controllable QDs will be obtained. Recently, our group prepared PbS QD-doped glass fiber directly by the melt-extraction method combined with moderate heat-treatment schedule. The size and distribution of PbS QDs in 


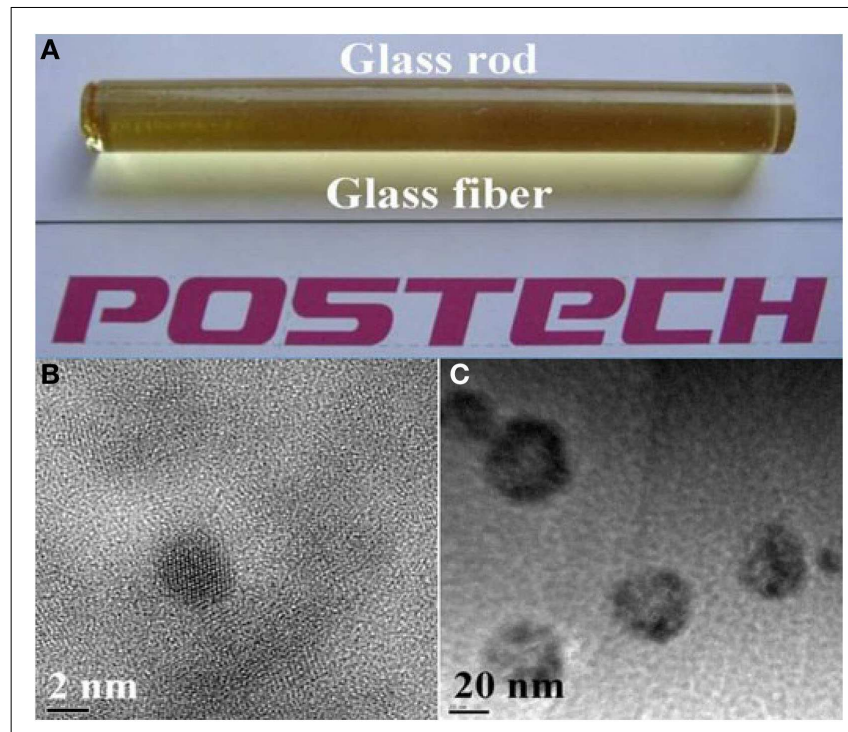

FIGURE 22 | Digital photographs of (A) the as-prepared glass preform and the obtained fiber. (B,C) TEM images of PbS nanocrystals formed during fiber-drawing process (Bhardwaj et al., 2012).

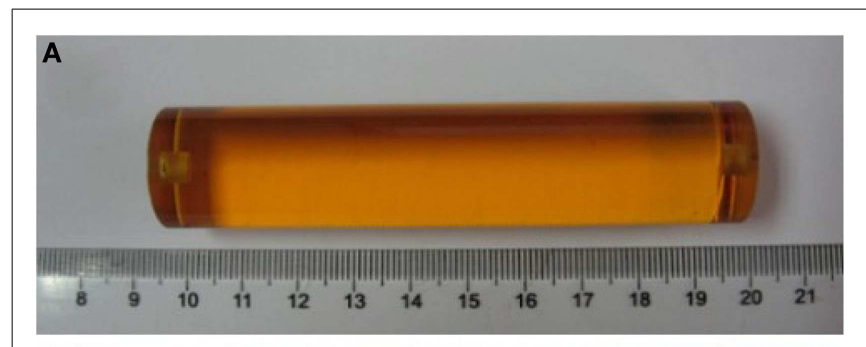

B

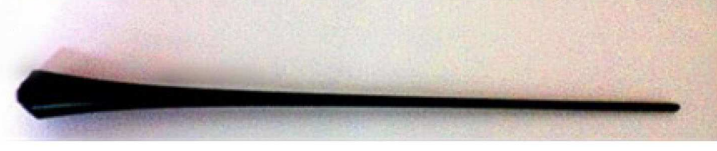

FIGURE 23 | (A) Digital photograph of optical fiber preform for the drawing of PbS QD-doped glass fiber. (B) Digital photograph of glass preform tail after fiber-drawing process.

fiber can be controlled by glass composition and heat-treatment schedule. Figure 24A illustrated the digital photographs of $\mathrm{PbS}$ QDs-doped glass fiber before and after heat-treatment at different schedules. The color of PbS QDs-doped glass fiber deepens when the heat-treatment temperature rises due to the controllable formation of PbS QDs in fiber after heat-treatment. From Figure 24B, it can be deduced that intense tunable NIR emissions were obtained in the above $\mathrm{PbS}$ QDs-doped glass fiber prepared by the melt-extraction method.

Herein, liquid-core fiber is the most convenient protocol to synthesis QDs with excellent performance. However, for further application, QDs must be incorporated into various bulk matrixes and the fiber core substrate materials have a lot of restrictions. Directly drawing fiber preform is a simple way to controllably fabricate QD-doped glass fiber, but secondary growth of QD is difficult to control. Melt-extraction method is prior to synthesize a QD-doped fiber due to the controllable size and distribution of QDs in various glass matrixes, which probably need a further in-depth research in the future. The above results illustrated that the feasibility to prepare the QD-doped glass fiber from bulk PbS QD-doped glass. And in our next step, we will develop several new types of fiber-drawing techniques to prepare all-solid-state QDs-doped glass fiber with whole core/cladding structure.

\section{CONCLUSION AND PROSPECTS ACHIEVEMENT}

Both absorption and PL of lead chalcogenide QDs can be tuned within the NIR wavelength range, covering the most important second and third optical communication windows (Chao and Jong, 2013). And the fluorescence peak positions can be controlled by the size and distribution of the QDs (Liu et al., 2009).

Our recent studies have confirmed the optical amplification at $\sim 1330$ and $\sim 1550 \mathrm{~nm}$ windows of PbS QDs-doped glasses (Guoping et al., 2011). By tuning the QDs size, distribution, and PL properties, optical amplification was achieved at both 1330 and $1550 \mathrm{~nm}$ windows simultaneously in single PbS QD-doped glass, which is vital for their potential application as tunable broadband optical amplifiers and lasers (Chao et al., 2008).

Surplus high-speed optical switch, optical storage, optical computing devices, frequency multiplier can be prepared with semiconductor QD-doped glass, which have large third-order nonlinear polarizability and quick response time, and can be used for the determination of molecular non-linear electric polarization.

To apply QDs-doped glass as the gain medium of fiber amplifier and laser device, a lot of studies about QD-doped optical fiber have been reported. Cheng and Zhang proposed a theoretical PbSe fiber amplifier with bandwidth about $50 \mathrm{~nm}$, by solving light-propagation equations and rate equations (Cheng and Zhang, 2007). Furthermore, they proposed a multi-QD codoped fiber amplifier, which has an ultra-broad bandwidth over $120 \mathrm{~nm}$ (Cheng, 2008). Jiang et al. reported a numerical model of a QD-doped fiber amplifier by a two-level model (Chun, 2009). From the simulated results, we know that the QD-doped fiber had ultra-broad gain spectra in the range 1200-1620 nm upon 1200$\mathrm{nm}$ pump. Cheng et al. reported $\mathrm{PbSe} Q \mathrm{QDFL}$ with a ring resonator using colloidal PbSe QDs as gain media in the background of UV curing adhesive (Cheng et al., 2013). Upon 980-nm pumping, a clear exciting threshold was recorded, and continuous yet stable laser oscillation at $1550 \mathrm{~nm}$ was observed for the first time. The results above all showed that the QDs-doped optical fiber have a vital promising application as the gain medium of NIR broadband fiber amplifiers and tunable fiber lasers.

\section{EXISTING PROBLEMS AND PROSPECTS}

The overlap of QDs absorption peak and PL peak (small stokes shift)

When a system (molecule or atom) absorbs a photon, it gains energy and activates into an excited state. One way for the system to relax is to emit a photon, thus releasing its energy (another way would be the transformation of heat energy). When the emitted photon has less energy than the absorbed photon, this energy 

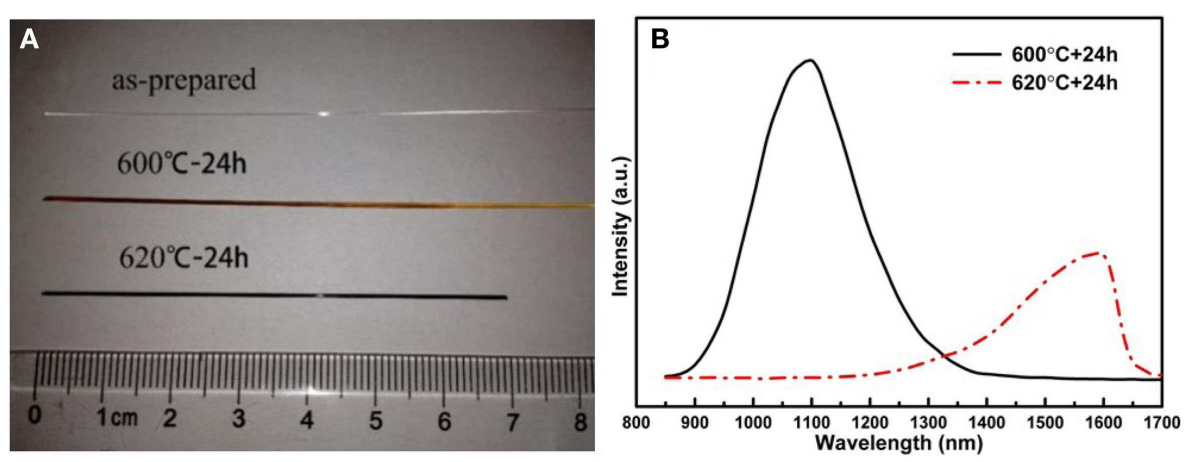

FIGURE 24 | (A) Digital photographs of PbS QD-doped glass fiber before and after heat-treatment at 600 and $620^{\circ} \mathrm{C}$ for $24 \mathrm{~h}$. (B) PL spectra of PbS QD-doped glass fiber after heat-treatment at different schedules.

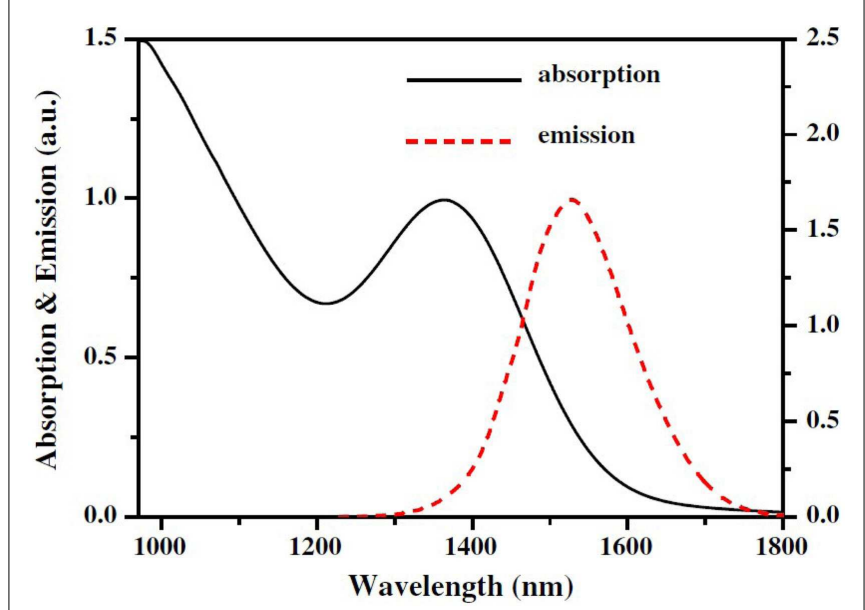

FIGURE 25 |The absorption and PL spectra of QDs, which illustrated a Stokes shift (Guo et al., 2012).

difference is the Stokes shift (Figure 25). With the decreasing of Stokes shift, the overlap of absorption and fluorescence spectrum increases, leading to the self-absorption which reduces the fluorescence efficiency. In general, a big Stokes shift is desirable to elevate emission efficiency within the interesting waveband (Cheng et al., 2011). Blue- or red-shift will occur by changing the size and distribution of QDs, with which the Stokes shift can be adjusted (Silva et al., 2006b). As the size of QDs increases, the Stokes shift will decrease (Cheng et al., 2011). This phenomenon was also reported in literature (Silva et al., 2003), which can be explained by the Kubo theory (Yu and Peng, 2002). The level spacing as a function of particle diameter can be expressed as follows (Kubo, 1962):

$$
\delta=\frac{4 \mathrm{E}_{F}}{3 N} \propto V^{-1}
$$

where $\delta$ is the level spacing, $N$ is the total number of conduction electrons of a nanoparticle, $V$ is the volume of a nanoparticle, and $E_{\mathrm{F}}$ is the Fermi level.

Through the above analysis, it can be deduced that the key to solve the problem of overlapping is to control the size and distribution of QDs. One can improve synthetic method of QDs, continuously explore new synthetic techniques to realize the controllable growth of QDs. By this, people can control the size and distribution of QD as required. In recent years, organic synthesis have been improved, and successfully prepared various bivariate and multivariate semiconductor QDs (such as: CdSe, PbS, InP, $\mathrm{ZnSe}, \mathrm{ZnxCd}_{1-\mathrm{x}} \mathrm{Se}, \mathrm{CdTe}_{\mathrm{x}} \mathrm{Se}_{1-\mathrm{x}}$, and so forth), realizing the controllable synthesis in size, morphology, composition, and properties of QD basically (Murray et al., 1993; Peng and Peng, 2001b; Yu and Peng, 2002).

Besides temperature, new fields including femtosecond laser, electrochemical synthesis, and magnetic field induction may also be utilized to tuning the distribution and growth of QDs in glass substrate. Stone et al. pointed out that the ferroelectric single crystal $\mathrm{LaBGeO}_{5}$ can controllably grow directly in $25 \mathrm{La}_{2} \mathrm{O}_{5}-25 \mathrm{~B}_{2} \mathrm{O}_{3}-$ $50 \mathrm{GeO}_{2}$ (mole percentage) glass by femtosecond laser irradiation (Stone et al., 2009). Later, Fan et al. also use femtosecond laser to control the micron-region formation of PbS QDs inside the glass. These fully prove that ultrafast laser radiation is one of the most effective ways to induce the controllable QDs formation in glass matrix (Fan et al., 2012). Now many scholars focus on finding out the effective laser radiation parameters to control crystallization behavior.

In summary, the size and distribution of the QDs can be designed to get the requiring Stokes shift, thereby weakening the overlap and self-absorption to further improve the QDs' quantum yield.

\section{Under the laser irradiation, photodarkening may be occurred in QDs-doped glass. The excessive ions (such as Pb and S elements for PbS QDs) in glass matrix will be deposited in the generated QDs during or after the process of laser irradiation, thus increasing the size of QDs, and weakening quantum confinement effect}

The key to solve this problem is to control the size of QDs, and they will not grow up in the role of light (Lagatsky et al., 2004). As is mentioned above, QD-doped glass and fiber can be prepared by composite approach of the QD nanocrystals with glass. Based on this method, QDs nanocrystals can be further cladding, their surface were passivation. And then they were compounded with glass, no any excessive ions (such as $\mathrm{Pb}$ and $\mathrm{S}$ elements for $\mathrm{PbS}$ 
QDs) existed in glass matrix. This makes the resistance of QDs growth much bigger, harder to grow when influenced by external field conditions.

A stable and widely covered way is growing inorganic material shell on the surface of QDs, forming core/shell structure. Inorganic material shell with the same clan system can grow on the surface of QD's nuclear epitaxial, it will be more insulation than semiconductor, effectively protect the carrier at the inside of the light. Among them, the $\mathrm{ZnS}$ shell can coat in the nuclear of CdSe (both are II-VI), which is the most common core/shell structure. The research about them is the thorough currently, and they have been widely used in various applications such as subsequent biological, photoelectric fields (Colvin et al., 1994; Bruchez et al., 1998).

\section{Secondary growth of QDs during the optical fiber-drawing process}

When drawing PbS QD-doped optical fiber from the preform, due to the excessive $\mathrm{Pb}$ and $\mathrm{S}$ elements in glass matrix, the secondary growth of QDs will occur via ion-migration mechanism under the driving force for the rise of temperature, so that the size of QDs is hard to control. Therefore, confinement effect is difficult to play since QDs easily grow larger than the exciton Bohr radius.

As is mentioned above, we can obtain fiber by melt-extraction method, and its advantage is easy to control the size of QD. But it is only used to prepare bare fiber. To obtain optical fiber that can be used for practical application, we must modify its fiber-drawing process, making direct access to prepare whole QD-doped glass fiber containing outer cladding layer.

Based on the successful fabrication of luminescent PbS QDdoped bare fibers by melt-extraction method mentioned above, a traditional type of fiber-drawing technology - double crucible method is expected to directly fabricate optical fiber containing outer cladding layer from glass melt. Sanghera et al. have fabricated single mode optical fibers using the double crucible technique, and that double crucible process enables adjustment of the core/clad diameter ratio during fiber drawing by independent pressure control above each melt (Sanghera et al., 2008). The mechanical property of glass fibers fabricated by double crucible process is better (Dianov et al., 1990). These all confirmed the feasibility of double crucible technique. After drawing by double crucible process, and then heat-treatment, moderate size of QDs can be obtained in fiber by controlling the heat-treatment schedule.

By composite approach of the QDs and glass, it is also expected to prepare QD-doped glass and fiber. The QDs should be controllably synthesized firstly, and then composited with the substrate glass together through co-melting technique (Chai et al., 2012), which can be further drawn into optical fiber by controlling the reasonable temperature and melting state. During the composite approach, there are no residual $\mathrm{Pb}$ and $\mathrm{S}$ elements in $\mathrm{PbS}$ QD-doped glass, and the QDs can only grow through the Oswald ripening process. Since the resistance of QDs growth via Oswald ripening process is much bigger than the above ionmigration force of secondary growth, it is much more difficult for the secondary growth of QDs by composite approach during the fiber-drawing process. In our previous work, we have successfully synthesized YAG nanocrystals, and making them recombined with substrate tellurate glass by novel co-melting technology.
Intense $2.7 \mu \mathrm{m}$ emission was obtained in the YAG: $\mathrm{Er}^{3+}, \mathrm{Tm}^{3+}$ nanocrystals-tellurate glass composites (Chai et al., 2012). Those works have opened a bright future work to fabricate high-quality QDs-doped glass fibers and devices.

\section{ACKNOWLEDGMENTS}

This work was supported by the National Natural Science Foundation of China (61475047, 51102096), the Guangdong Natural Science Foundation for Distinguished Young Scholars, and the project of Guangzhou Pearl River Science and Technology New Star (2014J2200083).

\section{REFERENCES}

Arnold, G. W. (1973). Ion implantation effects in noncrystallization SiO2. IEEE Trans. Nucl. Sci. 20, 220-223. doi:10.1109/TNS.1973.4327397

Arnold, G. W. (1975). Near-surface nucleation and crystallization of an ionimplanted lithia-alumina-silica glass. J. Appl. Phys. 46, 4466-4473. doi:10.1063/ 1.321422

Bahnemann, D., Henglein, A., Lilie, J., and Spanhel, L. (1984). Flash photolysis observation of the absorption spectra of trapped positive holes and electrons in colloidal TiO2. J. Phys. Chem. 88, 709-711. doi:10.1021/j150648a018

Beenakker, C. W. J. (1991). Theory of Coulomb-blockade oscillations in the conductance of a quantum dot. Phys. Rev. B Condens. Matter 44, 1646-1656. doi:10.1103/PhysRevB.44.1646

Bhardwaj, A., Hreibi, A., Chao, L., Heo, J., Auguste, J., Blondy, J., et al. (2012). "PbS quantum dots doped glass fibers for optical applications," in Conference on Lasers and Electro-Optics (Piscataway, NJ: IEEE), CTh1G.1.

Borrelli, N. F., and Smith, D. W. (1994). Quantum confinement of PbS microcrystals in glass. J. Non Cryst. Solids 180, 25-31. doi:10.1016/0022-3093(94)90393-X

Bruchez, M., Moronne, M., Gin, P., Weiss, S., and Alivisatos, A. P. (1998). Semiconductor nanocrystals as fluorescent biological labels. Science 281, 2013-2016. doi: $10.1126 /$ science.281.5385.2013

Brus, L. (1991). Quantum crystallites and nonlinear optics. Appl. Phys. A A53, 465-474. doi:10.1007/BF00331535

Brus, L. E. (1984). Electron-electron and electron-hole interactions in small semiconductor crystallites: the size dependence of the lowest excited electronic state. J. Chem. Phys. 80, 4403-4409. doi:10.1063/1.447218

Carder, D. A., Markwitz, A., Reeves, R. J., Kennedy, J., and Fang, F. (2013). Atomic retention and near infrared photoluminescence from PbSe nanocrystals fabricated by sequential ion implantation and electron beam annealing. Nucl. Instrum. Methods B 307, 154-157. doi:10.1016/j.nimb.2012.12.078

Chai, G., Dong, G., Qiu, J., Zhang, Q., and Yang, Z. (2012). $2.7 \mu \mathrm{m}$ emission from transparent $\mathrm{Er}^{3+}, \mathrm{Tm}^{3+}$ codoped yttrium aluminum garnet $\left(\mathrm{Y}_{3} \mathrm{Al}_{5} \mathrm{O}_{12}\right)$ nanocrystals-tellurate glass composites by novel comelting technology. J. Phys Chem. C 116, 19941-19950. doi:10.1021/jp3052906

Chao, L., and Jong, H. (2013). Lead chalcogenide quantum dot-doped glasses for photonic devices. Int. J. Appl. Glass Sci. 4, 163-173. doi:10.1111/ijag.12032

Chao, L., and Jong, H. (2014). Nanocrystal formation in glasses controlled by rare earth ions. Int. J. Appl. Glass Sci. 5, 104-113. doi:10.1111/ijag.12074

Chao, L., Jong, H., Xianghua, Z., and Adam, J. L. (2008). Photoluminescence of PbS quantum dots embedded in glasses. J. Non Cryst. Solids 354, 618-623. doi:10.1016/j.jnoncrysol.2007.07.069

Cheng, C. (2008). A multiquantum-dot-doped fiber amplifier with characteristics of broadband, flat gain, and low noise. J. Lightwave Technol. 26, 1404-1410. doi:10.1109/JLT.2008.923641

Cheng, C., Bo, J., Yan, J., and Cheng, X. (2013). Experimental realization of a PbSe-quantum-dot doped fiber laser. IEEE Photon. Tech. Lett. 25, 572-575. doi:10.1109/LPT.2013.2243139

Cheng, C., Jiang, H., Ma, D., and Cheng, X. (2011). An optical fiber glass containing PbSe quantum dots. Opt. Commun. 284, 4491-4495. doi:10.1016/j.optcom.2011. 05.004

Cheng, C., and Zhang, H. (2007). Characteristics of bandwidth, gain and noise of a PbSe quantum dot-doped fiber amplifier. Opt. Commun. 277, 372-378. doi:10.1016/j.optcom.2007.05.027

Chun, J. (2009). Ultrabroadband gain characteristics of a quantum-dot-doped fiber amplifier. IEEE J. Sel. Top. Quant. Electron. 15, 140-144. doi:10.1109/JSTQE. 2008.2010267 
Colvin, V. L., Schlamp, M. C., and Allvisatos, A. P. (1994). Light-emitting diodes made from cadmium selenide nanocrystals and a semiconducting polymer. Nature 370, 354-357. doi:10.1038/370354a0

Derfus, A. M., Chan, W. C. W., and Bhatia, S. N. (2004). Probing the cytotoxicity of semiconductor quantum dots. Nano Lett. 4, 11-18. doi:10.1021/nl0347334

Dianov, E. M., Krasteva, V. M., and Plotnichenko, V. G. (1990). "Mechanical properties of chalcogenide glass optical fibers," in SPIE-The International Society for Optical Engineering, Vol. 1228 (Los Angeles, CA: SPIE), 92-100.

EerNisse, E. P. (1974). Compaction of ion-implanted fused silica. J. Appl. Phys. 45, 167-174. doi:10.1063/1.1662952

Efros, A. L., and Efros, A. L. (1982). Interband absorption of light in a semiconductor sphere. Sov. Phys. Semiconduct. 16, 772-775.

Ekimov, A. I., Efros, A. L., and Onushchenko, A. A. (1985). Quantum size effect in semiconductor microcrystals. Solid State Commun. 56, 921-924. doi:10.1016/ S0038-1098(85)80025-9

Fan, C., Poumellec, B., Lancry, M., He, X., Zeng, H., Erraji-Chahid, A., et al. (2012). Three-dimensional photoprecipitation of oriented $\mathrm{LiNbO}_{3}$-like crystals in silica-based glass with femtosecond laser irradiation. Opt. Lett. 37, 2955-2957. doi:10.1364/OL.37.002955

Feng, W., Wei, T., Li-Na, M., Wen-Ju, C., Gui-Lan, Z., Guo-Feng, Z., et al. (2008). Optical nonlinear properties of $\mathrm{CdSeS} / \mathrm{ZnS}$ core/shell quantum dots. Chinese Phys. Lett. 25, 1461-1464. doi:10.1088/0256-307X/25/4/080

Grundmann, M., Christen, J., Ledentsov, N. N., Bohrer, J., Bimberg, D., Ruvimov, S. S., et al. (1995). Ultranarrow luminescence lines from single quantum dots. Phys. Rev. Lett. 74, 4043-4046. doi:10.1103/PhysRevLett.74.4043

Guerreiro, P. T., Ten, S., Borrelli, N. F., Butty, J., Jabbour, G. E., and Peyghambarian, N. (1997). PbS quantum-dot doped grasses as saturable absorbers for mode locking of a Cr:forsterite laser. Appl. Phys. Lett. 71, 1595-1597. doi:10.1063/1.119843

Guo, H., Pang, F., Zeng, X., and Wang, T. (2012). PbS quantum dot fiber amplifier based on a tapered SMF fiber. Opt. Commun. 285, 3222-3227. doi:10.1016/j. optcom.2012.02.075

Guoping, D., Botao, W., Fangteng, Z., Liaolin, Z., Mingying, P., Dongdan, C., et al. (2011). Broadband near-infrared luminescence and tunable optical amplification around $1.55 \mu \mathrm{m}$ and $1.33 \mu \mathrm{m}$ of PbS quantum dots in glasses. J. Alloys Compd. 509, 9335-9339. doi:10.1016/j.jallcom.2011.07.030

Guoping, D., Guobo, W., Shaohua, F., Fangteng, Z., Yuanhao, Z., Botao, W., et al. (2014). Formation, near-infrared luminescence and multi-wavelength optical amplification of PbS quantum dot-embedded silicate glasses. J. Non Cryst. Solids 383, 192-195. doi:10.1016/j.jnoncrysol.2013.04.010

Heck, M. J. R., Bente, E., Smalbrugge, B., Oei, Y. S., Smit, M. K., Anantathanasarn, S., et al. (2007). Observation of Q-switching and mode-locking in two-section InAs/InP (100) quantum dot lasers around $1.55 \mu \mathrm{m}$. Opt. Express 15, 16292-16301. doi:10.1364/OE.15.016292

Heinrichsdorff, F., Mao, M. H., Kirstaedter, N., Krost, A., Bimberg, D., Kosogov, A. O., et al. (1997). Room-temperature continuous-wave lasing from stacked InAs/GaAs quantum dots grown by metalorganic chemical vapor deposition. Appl. Phys. Lett 71, 22-24. doi:10.1063/1.120556

Henglein, A. (1988). Mechanism of reactions on colloidal microelectrodes and size quantization effects. Top. Curr. Chem. 143, 113-180. doi:10.1007/BFb0018073

Heo, J., and Liu, C. (2007). Pbs quantum-dots in glass matrix for universal fiberoptic amplifier. J. Mater. Sci Mater. Electron. 18, S135-S139. doi:10.1007/s10854007-9172-1

Hines, M. A., and Scholes, G. D. (2003). Colloidal PbS nanocrystals with size-tunable near-infrared emission: observation of post-synthesis self-narrowing of the particle size distribution. Adv. Mater. 15, 1844-1849. doi:10.1002/adma.200305395

Hoogland, S., Sukhovatkin, V., Howard, I., Cauchi, S., Levina, L., and Sargent, E. H. (2006). A solution-processed $1.53 \mu \mathrm{m}$ quantum dot laser with temperatureinvariant emission wavelength. Opt. Express 14, 3273-3281. doi:10.1364/OE.14. 003273

Hreibi, A., Gérôme, F., Auguste, J.-L., Dai, Q., Yu, W. W., and Blondy, J.-M. (2010). "PbSe quantum dots liquid-core fiber," in Lasers and Electro-Optics (CLEO) and Quantum Electronics and Laser Science Conference (QELS) (Piscataway, NJ: IEEE), $1-2$.

Huang, W., Chi, Y. Z., Wang, X., Zhou, S. F., Wang, L., Wu, E., et al. (2008). Tunable infrared luminescence and optical amplification in $\mathrm{PbS}$ doped glasses. Chinese Phys. Lett. 25, 2518-2520. doi:10.1088/0256-307X/25/7/051

Huang, X. J., Wu G., Ye, S., Wu, B., Dong, G., and Qiu, J. (in press). The effect of $\mathrm{ZnS} / \mathrm{ZnO}$ on the formation, luminescence and optical amplification of $\mathrm{PbS}$ quantum dots doped glasses. Sci. Adv. Mater. doi:10.1166/sam.2015.2312
Jacob, G. J., Barbosa, L. C., and Cesar, C. L. (2005). "Tellurite glass optical fiber doped with PbTe quantum dots," in SPIE: Quantum Dots, Nanoparticles, and Nonoclusters II, Vol. 5734 (Bellingham, WA: SPIE-The International Society for Optical Engineering), 124-129.

Jain, R. K., and Lind, R. C. (1983). Degenerate four-wave mixing in semiconductordoped glasses. J. Opt. Soc. Am. 73, 647-653. doi:10.1364/JOSA.73.000647

Kellermann, G., Craievich, A. F., Barbosa, L. C., and Alves, O. L. (2001). Nucleation and growth of $\mathrm{CdTe}_{1-\mathrm{x}} \mathrm{S}_{\mathrm{X}}$ nanocrystals embedded in a borosilicate glass. Effects of sulfur content and two-step thermal annealing. J. Non Cryst. Solids 293, 517-526. doi:10.1016/S0022-3093(01)00769-4

Kim, S., Lim, Y. T., Soltesz, E. G., De Grand, A. M., Lee, J., Nakayama, A., et al. (2004). Near-infrared fluorescent type II quantum dots for sentinel lymph node mapping. Nat. Biotechnol. 22, 93-97. doi:10.1038/nbt920

Klimov, V. I., Mikhailovsky, A. A., Xu, S., Malko, A., Hollingsworth, J. A., Leatherdale, C. A., et al. (2000). Optical gain and stimulated emission in nanocrystal quantum dots. Science 290, 314-317. doi:10.1126/science.290.5490.314

Kolobkova, E. V., Lipovskii, A. A., and Petrikov, V. D. (2002). Fluorophosphate glasses containing PbSe quantum dots. Glass Phys. Chem. 28, 246-250. doi:10.1023/A: 1019966413445

Kormann, C., Bahnemann, D. W., and Hoffmann, M. R. (1988). Preparation and characterization of quantum-size titanium dioxide. J. Phys. Chem. 92, 5196-5201. doi:10.1021/j100329a027

Kubo, R. (1962). Electronic properties of metallic fine particles. I. J. Phys. Soc. Jpn. 17, 975-986. doi:10.1016/j.envres.2014.05.015

Kunets, V. P., Kulish, N. R., Strelchuk, V. V., Nazarov, A., Tkachenko, A. S., Lysenko, V. S., et al. (2004). CdSSe quantum dots: effect of the hydrogen RF plasma treatment on exciton luminescence. Physica E 22, 804-807. doi:10.1016/j.physe.2003. 09.037

Kuntz, M., Fiol, G., Laemmlin, M., Meuer, C., and Bimberg, D. (2007). Highspeed mode-locked quantum-dot lasers and optical amplifiers. Proc IEEE 95, 1767-1778. doi:10.1109/JPROC.2007.900949

Lagatsky, A. A., Leburn, C. G., Brown, C. T. A., Sibbett, W., Malyarevich, A. M., Savitski, V. G., et al. (2004). Passive mode locking of a $\mathrm{Cr}^{4+}$ : YAG laser by $\mathrm{PbS}$ quantum-dot-doped glass saturable absorber. Opt. Commun. 241, 449-454. doi:10.1016/j.optcom.2004.07.022

Lee, J. K., Tewell, C. R., Schulze, R. K., Nastasi, M., Hamby, D. W., Lucca, D. A., et al. (2005). Synthesis of $\mathrm{ZnO}$ nanocrystals by subsequent implantation of $\mathrm{Zn}$ and O species. Appl. Phys. Lett. 86, 183111. doi:10.1063/1.1906304

Liu, C., Kwon, Y. K., and Heo, J. (2009). Absorption and photoluminescence of PbS QDs in glasses. J. Non Cryst. Solids 355, 1880-1883. doi:10.1016/j.jnoncrysol. 2009.04.045

Malyarevich, A. M., Denisov, I. A., Savitsky, V. G., Yumashev, K. V., and Lipovskii, A. A. (2000). Glass doped with $\mathrm{PbS}$ quantum dots for passive Q switching of a 1.54 $\mu \mathrm{m}$ laser. Appl. Opt. 39, 4345-4347. doi:10.1364/AO.39.004345

Manna, L., Scher, E. C., and Alivisatos, A. P. (2000). Synthesis of soluble and processable rod-, arrow-, teardrop-, and tetrapod-shaped CdSe nanocrystals. J. Am. Chem. Soc. 122, 12700-12706. doi:10.1021/ja003055+

Mashford, B., Baldauf, J., Tich-Lam, N., Funston, A. M., and Mulvaney, P. (2011). Synthesis of quantum dot doped chalcogenide glasses via sol-gel processing. J. Appl. Phys. 109, 094305. doi:10.1063/1.3579442

Mattern, P. L., Thomas, G. J., and Baure, W. (1976). Hydrogen and helium implantation in vitreous silica. J. Vac. Sci. Technol. 13, 430-436. doi:10.1116/1.568938

Meldrum, A., Boatner, L. A., and White, C. W. (2001). Nanocomposites formed by ion implantation: recent developments and future opportunities. Nucl. Instrum. Methods B 178, 7-16. doi:10.1016/S0168-583X(00)00501-2

Melekhin, V. G., Kolobkova, E. V., Lipovskii, A. A., Petrikov, V. D., Malyarevich, A. M., and Savitsky, V. G. (2008). Fluorophosphate glasses doped with PbSe quantum dots and their nonlinear optical characteristics. Glass Phys. Chem. 34, 351-355. doi:10.1134/S1087659608040020

Michalet, X., Pinaud, F. F., Bentolila, L. A., Tsay, J. M., Doose, S., Li, J. J., et al. (2005). Quantum dots for live cells, in vivo imaging, and diagnostics. Science 307, 538-544. doi:10.1126/science.1104274

Miller, D. A. B., Chemla, D. S., and Schmitt-Rink, S. (1988). Electroabsorption of highly confined systems: theory of the quantum-confined Franz-Keldysh effect in semiconductor quantum wires and dots. Appl. Phys. Lett. 52, 2154-2156. doi:10.1063/1.99562

Mirin, R. P., Ibbetson, J. P., Nishi, K., Gossard, A. C., and Bowers, J. E. (1995). 1.3 mum photoluminescence from InGaAs quantum dots on GaAs. Appl. Phys. Lett. 67, 3795-3797. doi:10.1063/1.115386 
Mukai, K., Ohtsuka, N., and Sugawara, N. (1997). High photoluminescence efficiency of InGaAs/GaAs quantum dots self-formed by atomic layer epitaxy technique. Appl. Phys. Lett. 70, 2416-2418. doi:10.1063/1.118889

Murray, C. B., Norris, D. J., and Bawendi, M. G. (1993). Synthesis and characterization of nearly monodisperse $\mathrm{CdE}$ ( $\mathrm{E}=$ sulfur, selenium, tellurium $)$ semiconductor nanocrystallites. J. Am. Chem. Soc. 115, 8706-8715. doi:10.1021/ ja00072a025

O'Connor, E., O'Riordan, A., Doyle, H., Moynihan, S., Cuddihy, A., and Redmond, G. (2005). Near-infrared electroluminescent devices based on colloidal HgTe quantum dot arrays. Appl. Phys. Lett. 86:201114. doi:10.1063/1.1928321

Olkhovets, A., Hsu, R. C., Lipovskii, A., and Wise, F. W. (1998). Size-dependent temperature variation of the energy gap in lead-salt quantum dots. Phys. Rev. Lett. 81, 3539-3542. doi:10.1103/PhysRevLett.81.3539

Pang, F., Sun, X., Guo, H., Yan, J., Wang, J., Zeng, X., et al. (2010). A PbS quantum dots fiber amplifier excited by evanescent wave. Opt. Express 18, 14024-14030. doi:10.1364/OE.18.014024

Peng, X. G., Wickham, J., and Alivisatos, A. P. (1998). Kinetics of II-VI and III-V colloidal semiconductor nanocrystal growth: "focusing" of size distributions. J. Am. Chem. Soc. 120, 5343-5344. doi:10.1021/ja9805425

Peng, Z. A., and Peng, X. G. (2001a). Mechanisms of the shape evolution of CdSe nanocrystals. J. Am. Chem. Soc. 123, 1389-1395. doi:10.1021/ja0027766

Peng, Z. A., and Peng, X. G. (2001b). Formation of high-quality CdTe, CdSe, and CdS nanocrystals using CdO as precursor. J. Am. Chem. Soc. 123, 183-184. doi:10.1021/ja003633m

Planelles-Arago, J., Julian-Lopez, B., Cordoncillo, E., Escribano, P., Pelle, F., Viana, B., et al. (2008). Lanthanide doped $\mathrm{ZnS}$ quantum dots dispersed in silica glasses: an easy one pot sol-gel synthesis for obtaining novel photonic materials. J. Mater. Chem. 18, 5193-5199. doi:10.1039/b809254k

Primak, W., and Kampwith, R. (1968). The radiation compaction of vitreous silica. J. Appl. Phys. 39, 5651-5653. doi:10.1063/1.1656029

Rajh, T., Vucemilovic, M. I., Dimitrijevic, N. M., Micic, O. I., and Nozik, A. J. (1988). Size quantization of colloidal semiconductor particles in silicate glasses. Chem. Phys. Lett. 143, 305-308.

Rogach, A. L., Franzl, T., Klar, T. A., Feldmann, J., Gaponik, N., Lesnyak, V., et al. (2007). Aqueous synthesis of thiol-capped CdTe nanocrystals: state-of-the-art. J. Phys. Chem. C 111, 14628-14637. doi:10.1021/jp072463y

Salavati-Niasari, M., Sobhani, A., and Davar, F. (2010). Synthesis of star-shaped $\mathrm{PbS}$ nanocrystals using single-source precursor. J. Alloys Compd. 507, 77-83. doi:10.1016/j.jallcom.2010.06.062

Sanghera, J. S., Florea, C. M., Shaw, L. B., Pureza, P., Nguyen, V. Q., Bashkansky, M., et al. (2008). Non-linear properties of chalcogenide glasses and fibers. J. Non Cryst. Solids 354, 462-467. doi:10.1016/j.jnoncrysol.2007.06.104

Sargent, E. H. (2005). Infrared quantum dots. Adv. Mater. 17, 515-522. doi:10.1002/ adma.200401552

Savitski, V. G., Posnov, N. N., Prokoshin, P. V., Malyarevich, A. M., Yumashev, K. V., Demchuk, M. I., et al. (2002). PbS-doped phosphate glasses saturable absorbers for 1.3-mu m neodymium lasers. Appl. Phys. B 75, 841-846. doi:10.1007/s00340-002-0951-3

Schaller, R. D., Petruska, M. A., and Klimov, V. I. (2003). Tunable near-infrared optical gain and amplified spontaneous emission using PbSe nanocrystals. J. Phys. Chem. B 107, 13765-13768. doi:10.1021/jp0311660

Silva, R. S., Monte, A. F. G., Morais, P. C., Alcalde, A. M., Qu, F., and Dantasc, N. O. (2006a). Synthesis and characterization of $\mathrm{PbS}$ quantum dots embedded in oxide glass. Braz. J. Phys. 36, 394-396. doi:10.1590/S0103-97332006000300043

Silva, R. S., Morais, P. C., Alcalde, A. M., Qu, F., Monte, A. F. G., and Dantas, N. O. (2006b). Optical properties of PbSe quantum dots embedded in oxide glass. J. Non Cryst. Solids 352, 3522-3524. doi:10.1016/j.jnoncrysol.2006.03.114

Silva, R. S., Qu, F., Alcalde, A. M., and Dantas, N. O. (2003). Atomic force microscopy and optical characterization of $\mathrm{PbS}$ quantum dots grown in glass matrix. Microelectron. J. 34, 647-649. doi:10.1016/S0026-2692(03)00083-1

Sohila, S., Rajalakshmi, M., Ghosh, C., Arora, A. K., and Muthamizhchelvan, C. (2011). Optical and Raman scattering studies on SnS nanoparticles. J. Alloys Compd. 509, 5843-5847. doi:10.1016/j.jallcom.2011.02.141

Spanhel, L., Haase, M., Weller, H., and Henglein, A. (1987). Photochemistry of colloidal semiconductors. 20. Surface modification and stability of strong luminescing CdS particles. J. Am. Chem. Soc. 109, 5649-5655. doi:10.1021/ ja00253a015

Steiner, D., Katz, D., Millo, O., Aharoni, A., Kan, S., Mokari, T., et al. (2004). Zerodimensional and quasi one-dimensional effects in semiconductor nanorods. Nano Lett. 4, 1073-1077. doi:10.1039/b9nr00282k

Stone, A., Sakakura, M., Shimotsuma, Y., Stone, G., Gupta, P., Miura, K., et al. (2009). Directionally controlled 3D ferroelectric single crystal growth in $\mathrm{LaBGeO}_{5}$ glass by femtosecond laser irradiation. Opt. Express 17, 23284-23289. doi:10.1364/ OE.17.023284

Sukhovatkin, V., Musikhin, S., Gorelikov, I., Cauchi, S., Bakueva, L., Kumacheva, E., et al. (2005). Room-temperature amplified spontaneous emission at 1300 $\mathrm{nm}$ in solution-processed PbS quantum-dot films. Opt. Lett. 30, 171-173. doi:10.1364/OL.30.000171

Takagahara, T. (1987). Excitonic optical nonlinearity and exciton dynamics in semiconductor quantum dots. Phys. Rev. B Condens. Matter 36, 9293-9296. doi:10.1103/PhysRevB.36.9293

Tan, S. T., Chen, B. J., Sun, X. W., Fan, W. J., Kwok, H. S., Zhang, X. H., et al. (2005). Blueshift of optical band gap in $\mathrm{ZnO}$ thin films grown by metal-organic chemical-vapor deposition. J. Appl. Phys. 98, 0.013505. doi:10.1063/1.1940137

Thantu, N. (2005). Second harmonic generation and two-photon luminescence upconversion in glasses doped with $\mathrm{ZnSe}$ nanocrystalline quantum dots. J. Lumin. 111, 17-24. doi:10.1016/j.jlumin.2004.06.002

Weller, H., Koch, U., Gutierrez, M., and Henglein, A. (1984). Photochemistry of colloidal metal sulfides. 7. Absorption and fluorescence of extremely small $\mathrm{ZnS}$ particles (the world of the neglected dimensions). Ber. Bunsen-Ges. Phys. Chem. $88,649-655$.

Wise, F. W. (2000). Lead salt quantum dots: the limit of strong quantum confinement. Acc. Chem. Res. 33, 773-780. doi:10.1021/ar970220q

Wundke, K., Auxier, J., Schulzgen, A., Peyghambarian, N., and Borrelli, N. F. (1999). Room-temperature gain at $1.3 \mu \mathrm{m}$ in PbS-doped glasses. Appl. Phys. Lett. 75, 3060-3062. doi:10.1063/1.125230

Xiaogang, P., Manna, L., Weidong, Y., Wickham, J., Scher, E., Kadavanich, A., et al. (2000). Shape control of CdSe nanocrystals. Nature 404, 59-61. doi:10.1038/ 35003535

$\mathrm{Xu}, \mathrm{K}$., and Heo, J. (2014). Precipitation of PbS quantum dots in glasses by thermal diffusion of $\mathrm{Ag}^{+}$ions from silver pastes. J. Non Cryst. Solids 387, 76-78. doi:10.1016/j.jnoncrysol.2014.01.015

Yu, W. W., Falkner, J. C., Shih, B. S., and Colvin, V. L. (2004). Preparation and characterization of monodisperse PbSe semiconductor nanocrystals in a noncoordinating solvent. Chem. Mater. 16, 3318-3322. doi:10.1021/cm049476y

Yu, W. W., and Peng, X. G. (2002). Formation of high-quality CdS and other II-VI semiconductor nanocrystals in noncoordinating solvents: tunable reactivity of monomers. Angew. Chem. Int. Ed. 41, 2368-2371. doi:10.1002/15213773(20020703)41:13<2368::AID-ANIE2368>3.0.CO;2-G

Zhonghai, L., Minqiang, W., Lizhuo, W., Xiaohui, S., Yaohui, X., and Xi, Y. (2011). PbTe colloidal nanocrystals: synthesis, mechanism and infrared optical characteristics. J. Alloys Compd. 509, 5047-5049. doi:10.1016/j.jallcom.2011.02.028

Conflict of Interest Statement: The authors declare that the research was conducted in the absence of any commercial or financial relationships that could be construed as a potential conflict of interest.

Received: 04 January 2015; accepted: 07 February 2015; published online: 23 February 2015.

Citation: Dong G, Wang H, Chen G, Pan Q and Qiu J (2015) Quantum dotdoped glasses and fibers: fabrication and optical properties. Front. Mater. 2:13. doi: 10.3389/fmats.2015.00013

This article was submitted to Glass Science, a section of the journal Frontiers in Materials.

Copyright (c) 2015 Dong, Wang, Chen, Pan and Qiu. This is an open-access article distributed under the terms of the Creative Commons Attribution License (CC BY). The use, distribution or reproduction in other forums is permitted, provided the original author(s) or licensor are credited and that the original publication in this journal is cited, in accordance with accepted academic practice. No use, distribution or reproduction is permitted which does not comply with these terms. 